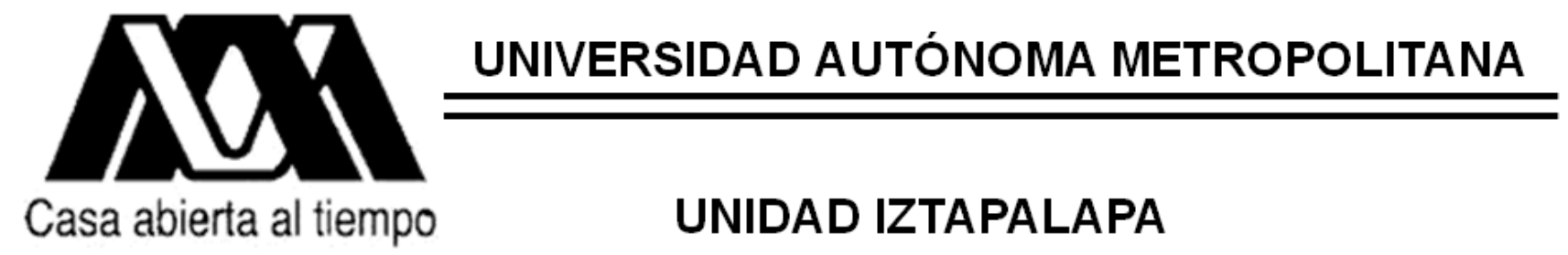

\title{
EFECTO DE LA ORIENTACIÓN Y CARACTERES DE LAS FLORES EN EL ÉXITO REPRODUCTIVO DE Pachycereus weberi EN LA REGIÓN DE TEHUACÁN-CUICATLÁN
}

\author{
TES IS \\ QUE PARA OBTENER EL GRADO ACADÉMICO DE \\ MAESTRA EN BIOLOGÍA \\ P R E S E T A \\ Biól. Esperanza Córdova Acosta
}

Director de tesis: Dr. Pedro Luis Valverde Padilla

Asesores: M. en C. Fernando Vite González

Dr. Alejandro Casas Fernández

MÉXICO, D.F.

MAYO, 2011. 
“La Maestría en Biología de la Universidad Autónoma Metropolitana pertenece al Padrón de Postgrados de Excelencia del CONACYT" 
El jurado designado por la

\section{División de Ciencias Biológicas y de la Salud}

de la Unidad Iztapalapa aprobó la tesis que presentó

Biól. Esperanza Córdova Acosta

El día 06 de mayo del 2011.

\section{Comité Tutoral}

Director: Dr. Pedro Luis Valverde Padilla

Asesor: M. en C. Fernando Vite González

Asesor: Dr. Alejandro Casas Fernández

Sinodal: Dr. José Alejandro Zavala Hurtado

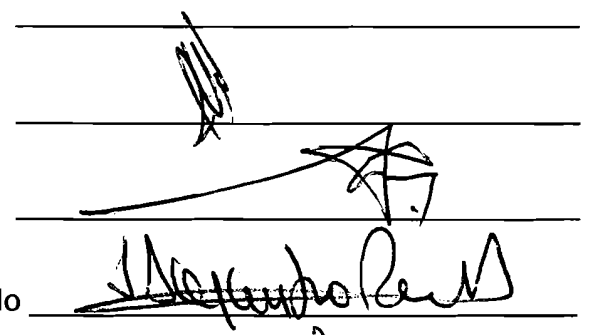

Sinodal: Dra. Maria del Carmen Mandujano Sánchez

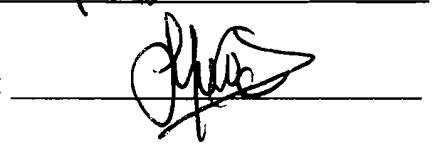


A mis dos estrellas que están en el cielo y que iluminan mi camino, mis abuelitas Amparo Natalia y María.

A mis padres Esperanza y Gregorio que han sido la columna vertebral de mi vida y la fuente de mi inspiración.

A mis hermanos Mario Rafael y María Gabriela que han sido la luz de mis ojos y la alegría de mi vida.

A mi bella sobrinita Andrea que es el sol que brilla en nuestras vidas.

A mis tíos José Isabel Acosta y Bonifacio Méndez Acosta por su protección y amor infinito.

A todas esas personas que de forma directa o indirecta han contribuido en enriquecer y mejorar el ser humano que soy. 


\section{AGRADECIMIENTOS}

Al Consejo Nacional de Ciencia y Tecnología (CONACyT) por el apoyo económico brindado para la realización de estos estudios de posgrado.

A la Universidad Autónoma Metropolitana por ser mí casa a lo largo de mi formación académica.

Agradezco de manera muy especial a mi Comité Tutoral: conformado por el Dr. Pedro Luis Valverde Padilla, a quien agradezco la dirección de este proyecto y por su apoyo incondicional en todo momento; al M. en C. Fernando Vite González por su paciencia infinita y por sus valiosos comentarios que permitieron enriquecer dicho trabajo y al Dr. Alejandro Casas Fernández por su excelente asesoramiento, su paciencia infinita y sus acertados consejos a lo largo de este trabajo.

Al Dr. José Alejandro Zavala Hurtado por aceptar ser parte del $\mathrm{H}$. jurado, por sus excelentes comentarios y sugerencias que permitieron mejorar este manuscrito.

A la Dra. María del Carmen Mandujano Sánchez por haber aceptado ser parte del jurado, por su tiempo y por sus valiosas aportaciones a este manuscrito.

Agradezco a los doctores Miguel Martínez Ramos, Horacio Paz y Francisco Espinosa su amistad, apoyo y conocimiento adquirido durante mi estancia en el Centro de Investigaciones en Ecosistemas (CIEco, UNAM).

A la Dra. Dulce Figueroa-Castro por su apoyo y asistencia en campo. 


\section{AGRADECIMIENTOS ESPECIALES}

Agradezco infinitamente a todos los pobladores y autoridades de San José Chichihualtepec, Oaxaca, por las facilidades brindadas para la realización del trabajo de campo en esta región.

¡Sin su valiosa colaboración, este trabajo hubiera sido imposible, mil gracias!

De manera muy especial, agradezco al Sr. Gregorio Mora y a su familia por su apoyo, hospitalidad y cariño con el cual siempre fuimos recibidos en su hogar, en más de una ocasión a lo largo de este estudio con el majestuoso "chico"

(Pachycereus weberi).

¡Gracias por esas amenas pláticas y por su amistad sincera!

Al Sr. Pedro Miranda Pacheco por su apoyo en campo al inicio de este proyecto, por su sabiduría, su valiosa amistad y por esa gran sonrisa con la que siempre nos recibe al Equipo de Ecología de Zonas Áridas de la UAM-I. ¡Gracias Don Peter!

Desde el fondo de mi corazón agradezco a mis cinco pilares y protectores académicos: Pedro Luis Valverde, Fernando Vite, Marco Aurelio Pérez, José Alejandro Zavala y Alejandro Casas su amistad, apoyo, cobijo y su cariño infinito en todo momento.

¡Cada uno de ustedes ha contribuido en ser lo que soy, mil gracias!

Agradezco de forma muy especial y particular a mis muy queridos amigos: José Alejandro Zavala por sus consejos, apoyo y respaldo incondicional cuando más lo he necesitado. Eres un gran bálsamo de luz en mi camino. Agradezco a la vida el 
haberte conocido y es un gran honor ser tu amiga. Gracias por brindarme tu mano y reír junto conmigo. ¡Eres una persona realmente excepcional en todos sentidos!

¡Mil gracias por ser y estar Ale!

A mi querido amigo Alejandro Casas por ser una personita especial y maravillosa. Eres un gran ser humano Casas y te mereces toda mi admiración por ser la persona que eres. Eres realmente "único" en toda la extensión de la palabra. Me siento muy afortunada de ser tu amiga y de haber aprendido mucho de ti y estoy segura que así seguirá siendo.

¡Gracias por hacerme sentir parte del "Equipo Casas"!

A mis "profesores consentidos" del Departamento de Biología de la UAM-I: Alejandro Zavala, Fernando Vite, Pedro Luis Valverde, Marco Aurelio Pérez, Gerardo López, Beatriz Rendón, Gilberto Hernández, Maricarmen Herrera, Angélica Martínez, Marú Fraile, entre otros más. Muchas gracias por los hermosos momentos vividos y las múltiples muestras de afecto hacia mi persona.

Agradezco de forma muy especial a Laurita Almaraz, Margarita, Maricela y Juanita personas invaluables del Departamento de Biología de la UAM-I.

¡Gracias por tantos años de cariño y amistad entrañable!

A todos mis compañeros y amigos de la generación 2008 de la Maestría en Biología por haberme dado la oportunidad de conocerlos y pasar momentos increíbles a su lado. Por su solidaridad y amistad siempre constantes. 
A mis inseparables amigas: Monse Jiménez, Amelia Cornejo, Evelyn Rosas y Paola Durante por brindarme su respaldo y consuelo cuando más lo necesite. Por los hermosos momentos y las locas aventuras emprendidas. Su amistad ha sido un gran tesoro que la vida me ha dado.

¡Gracias por existir en mi vida!

A mis queridos amigos Luis Alberto Bernal, David Bravo, Jesús Campos, Aileth Bazan y Jorge Orendain por ser mi apoyo constante y brindarme su cariño en todo momento.

A mis queridos amigos del "Equipo Casas" (Laboratorio de Ecología y Evolución de Recursos Vegetales, CIEco, UNAM): Faby, Sele, Susa, Dánae, Ana Isabel, Erandi, Nacho, América, Chalino, Xitlali, Juanita y Alex. Gracias a todos por brindarme su valiosa amistad, además de los consejos y sugerencias que me dieron para enriquecer este trabajo.

¡Me hicieron sentir como en casa!

\section{A mi familia}

De forma aún más especial, agradezco a mis padres su amor, esfuerzo y lucha constante. Gracias a ustedes aprendí el verdadero valor de la vida, la importancia de luchar por nuestros sueños y a valorar las personas que llegan a nuestra vida. Aprendí a estar en conexión con la madre tierra, amar y respetar la naturaleza como ¡Lo que soy es gracias a ustedes, los amo!

Agradezco a mis queridos hermanos Mario Rafael y María Gabriela por quererme como lo hacen, por los buenos momentos disfrutados y por la hermosa unión de solidaridad y amor que tenemos los tres. 
Agradezco a mi hermana María Gabriela el apoyo y la "presión diaria" que ejerció sobre mí para impulsarme a terminar este manuscrito.

Agradezco a mis tíos José Isabel Acosta y Bonifacio Méndez Acosta su cariño, amor y respaldo constante a lo largo de mi vida.

¡Ustedes son una pieza clave en mi formación como ser humano!

Agradezco a la vida la oportunidad de haber conocido a mis tíos Gaby y Gustavo Sánchez en este nuevo renacer de sus vidas. El tiempo nos dio la oportunidad de conocer a unos valiosos seres humanos.

¡Mil gracias!

Y gracias a todos aquellos amigos que he tenido la fortuna de conocer a lo largo de mi vida y que por causa del destino hemos tomado caminos distintos y emprendido nuevos retos.

¡Mil gracias por tanta felicidad, cariño, amistad y apoyo incondicional!

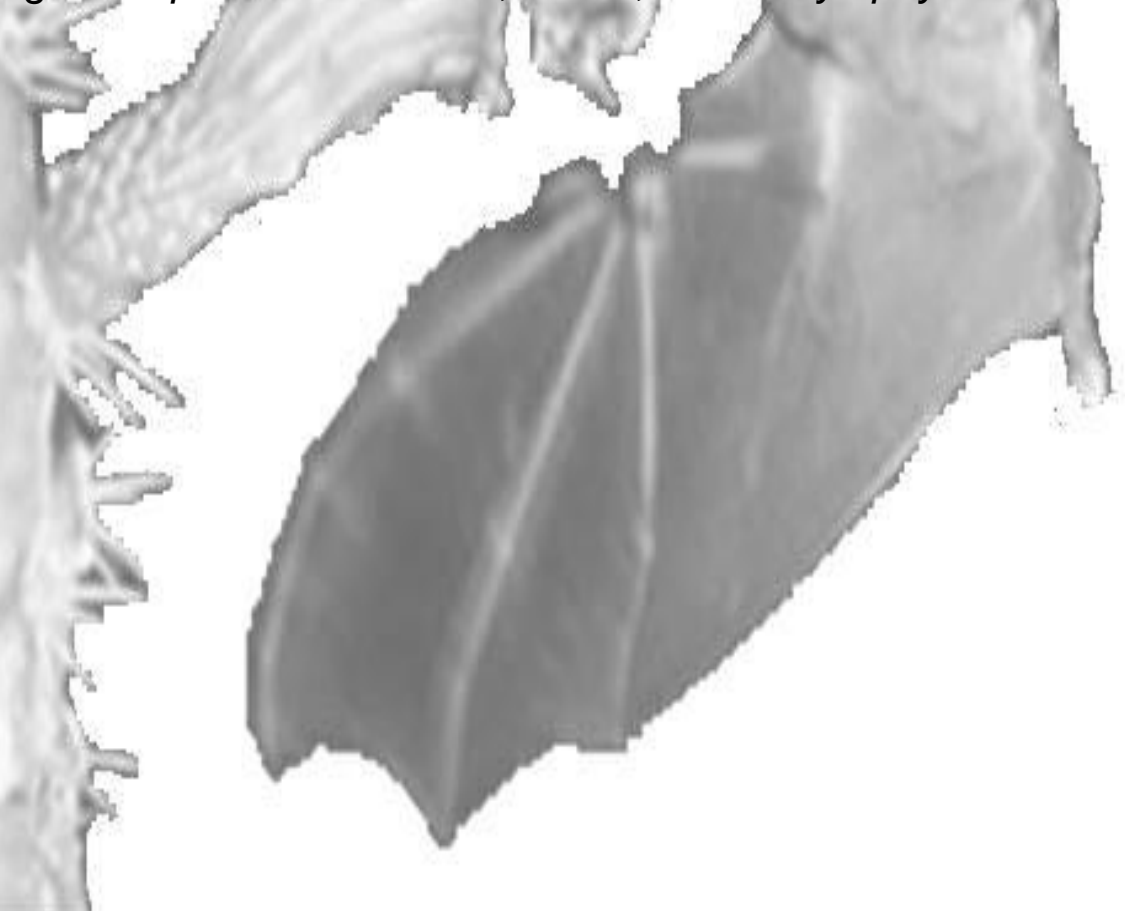


"Háblale al peyote con tu corazón, con tu pensamiento. Yel peyote verá tu corazón (...) $\Upsilon$ si tienes suerte, escucharás cosas y recibirás cosas que son invisibles para los demás, Pero que Dios te las da para que busques tu camino. Esta planta contiene el poder del espíritu" 


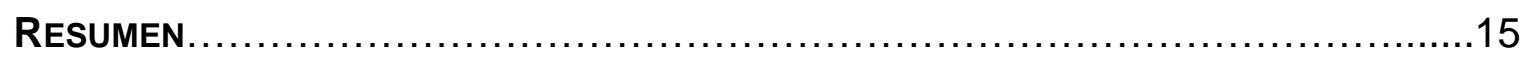

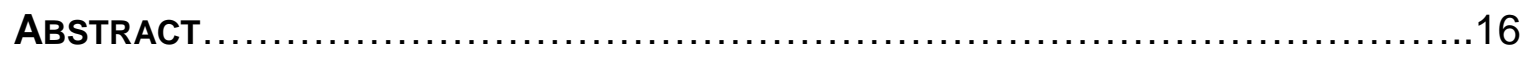

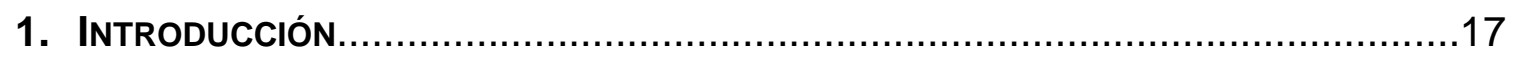

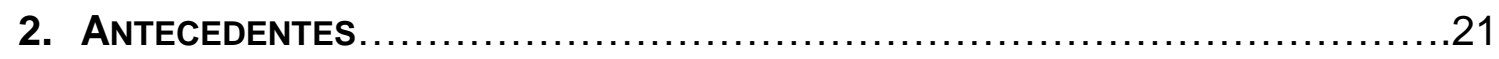

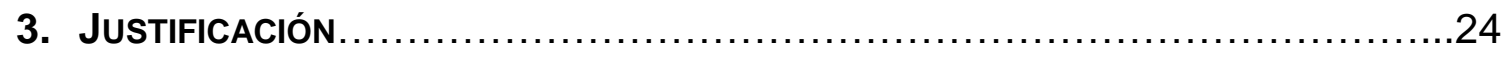

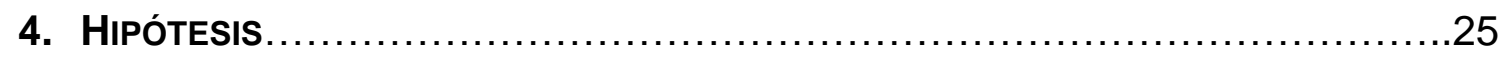

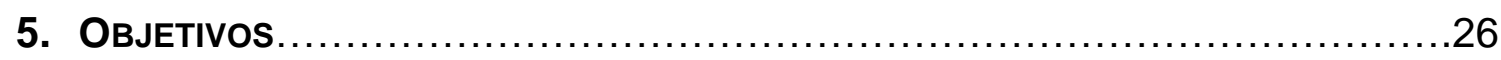

6. Materiales y mÉtodos

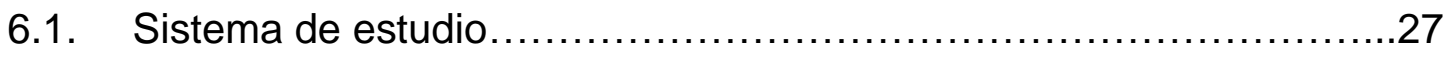

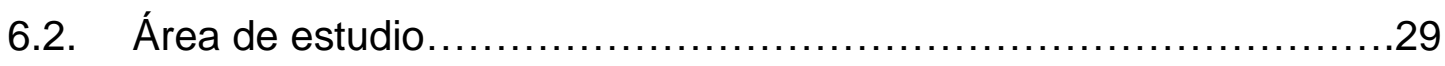

6.3. Determinación de la orientación preferencial de estructuras

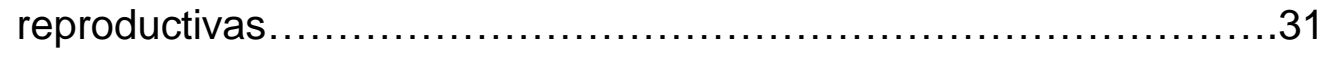

6.3.1. Registro de las condiciones microambientales: Temperatura y humedad relativa ...............................................

6.4. Medición de los caracteres florales y su relación con el éxito

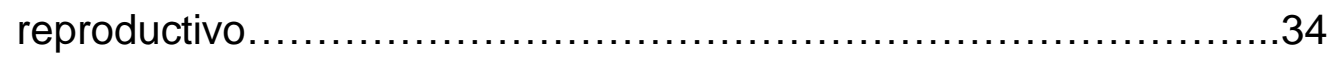

6.4.1. Experimento para evaluar el efecto del aroma como caracter floral y su relación con el éxito reproductivo.

\section{Resultados}

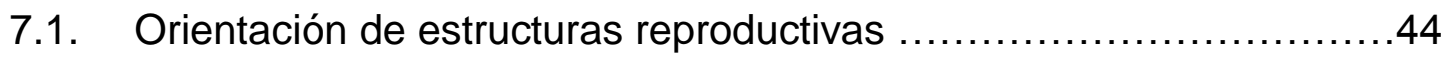

7.1.1. Registro de las condiciones microambientales: Temperatura y humedad relativa ................................................ 47

7.2. Variación de los caracteres florales y su relación con el éxito reproductivo.

7.2.1. Experimento para evaluar el efecto del aroma como carácter floral y su relación con el éxito reproductivo. 


\section{Discusión}

Orientación de estructuras reproductivas ................................54

Relación entre los caracteres florales asociados al síndrome de polinización quiropterófilo en Pachycereus weberi.

Efecto de la variación de los caracteres florales sobre el éxito reproductivo de $P$. weberi.................................................................59

Experimento para evaluar el efecto del aroma como carácter floral y su relación con el éxito reproductivo ............................................

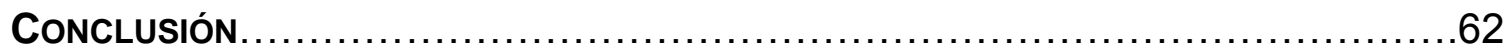

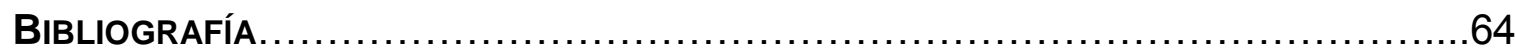


ÍNDICE DE FIGURAS

Figura 1. Sistema de estudio: Pachycereus weberi............................28

Figura 2. Mapa de las localidades de estudio....................................30

Figura 3. Área de cobertura de cada individuo dividida en sectores...............32

Figura 4. Conteo del número de costillas y estructuras reproductivas (sentido de las manecillas del reloj).............................................................

Figura 5. HOBO's colocados en la parte superior de una rama de $P$. weberi para registrar la temperatura y humedad relativa........................................34

Figura 6. Flores etiquetadas medidas in situ en San José Chichihualtepec,

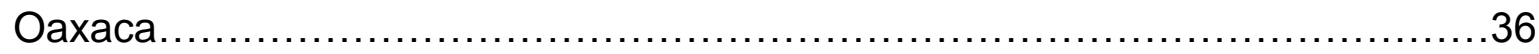

Figura 7. Medición de la longitud de una de las flores de $P$. weberi................37

Figura 8. Medición in situ del ángulo de inclinación en una de las flores de $P$.

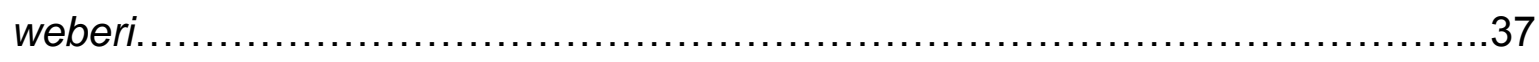

Figura 9. Flor etiquetada y embolsada para garantizar el posible fruto

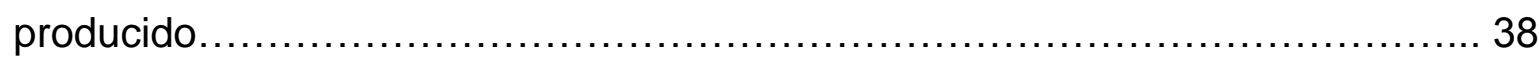

Figura 10. Fruto producido por una de las flores medidas in situ..................38

Figura 11. Tratamiento experimental con el "aroma aumentado" en un botón floral a "punto de abrir" en un individuo de $P$. weberi.

Figura 12. Tratamiento control en un botón floral "a punto de abrir" en un individuo de $P$. weberi.

Figura 13. Distribución de la frecuencia circular de estructuras reproductivas...45

Figura 14. Distribución de la frecuencia circular de yemas, flores y frutos de $P$. weberi. .46 
Figura 15. Marchas de temperatura y humedad relativa en los cuatro puntos cardinales.....

Figura 16. Relación entre la orientación y el diámetro de la corola de las flores de P. weberi. .50

Figura 17. Relación entre la orientación y el número de óvulos/ovario en botones colectados de $P$. weberi. 51

\section{ÍNDICE DE TABLAS}

Tabla 1. Estadísticos circulares básicos de la orientación de estructuras reproductivas.

Tabla 2. Resultado del análisis de regresión logística múltiple (modelo reducido) utilizado para evaluar el efecto de los caracteres florales medidos in situ $(n=59)$ sobre la proporción de frutos exitosos.

Tabla 3. Resultado del análisis de regresión múltiple (modelo reducido) utilizado para evaluar el efecto de los caracteres florales medidos in situ $(n=59)$ sobre el número de semillas.

Tabla 4. Pruebas de $t$-pareadas de una cola de la comparación entre tratamientos del experimento para evaluar el efecto del aroma en el éxito reproductivo .53 


\section{RESUMEN}

La distribución no aleatoria de estructuras reproductivas es un factor importante en el éxito de la producción de frutos y semillas en cactáceas, pues puede determinar condiciones microambientales favorables para el desarrollo y crecimiento de los botones, flores y frutos. Pachycereus weberi es un cacto columnar arborescente gigante, endémica de México, que se distribuye en los estados de Puebla, Oaxaca, Morelos, Guerrero e Hidalgo. Sus flores son hermafroditas, auto-incompatibles, presentan antesis predominantemente nocturna y son polinizadas principalmente por murciélagos. Este estudio analiza el efecto de la orientación y la variación de los caracteres florales asociados al síndrome de polinización quiropterófilo en el éxito reproductivo (proporción de frutos exitosos y proporción de semillas) de $P$. weberi en la Reserva de la Biosfera Tehuacán-Cuicatlán. Para determinar el patrón de orientación, se contaron las estructuras reproductivas en 42 individuos de $P$. weberi localizados en el km 73 de la carretera federal Tehuacán-Oaxaca. Se colocaron 12 dispositivos automatizados en tres individuos de esta especie para registrar la temperatura y humedad relativa en puntos con distinta orientación. La variación en orientación, diámetro de la corola, diámetro medio y basal, longitud y ángulo de inclinación de las flores y su relación con el éxito reproductivo se evaluó en 59 flores de $P$. weberi en San José Chichihualtepec, Oaxaca. Con los frutos y semillas producidos se estimó la proporción de frutos exitosos (fruit-set) y la proporción de semillas (seed-set), respectivamente. El número de óvulos/ovario se estimó en una muestra de 57 botones florales. Los análisis indican que $P$. weberi muestra un patrón de orientación sureño de sus estructuras reproductivas, el cual coincide con elevadas temperaturas durante el día y valores más altos de humedad relativa durante la noche. Estas condiciones benefician el desarrollo de las flores debido al incremento de las temperaturas alcanzadas por una orientación ecuatorial favoreciendo la actividad meristemática. La orientación y el diámetro medio de la flor (corola) son los caracteres florales que explican el éxito reproductivo de Pachycereus weberi, en términos de producción de frutos y semillas. Esto sugiere que las flores con mayor diámetro medio (una medida de su tamaño) y orientaciones sureñas son más exitosas en producir frutos y semillas. Los resultados del experimento sugieren que el aroma de las flores, es un carácter importante en el éxito reproductivo, pero las diferencias no fueron significativas. Esta información sugiere que la orientación de las flores y la variabilidad de los caracteres florales tienen efectos positivos en la polinización y en los eventos post-polinización. El presente estudio subraya la importancia de la selección de rasgos florales ejercida por los murciélagos y sus consecuencias en el éxito reproductivo en $P$. weberi. 


\section{Abstract}

The nonrandom distribution of reproductive structures in cacti is an important factor in the successful production of fruits and seeds, allowing to expose buds, flowers and fruits to appropriate micro-environmental conditions for their development and growth. Pachycereus weberi is a giant columnar arborescent cactus that is distributed in the states of Puebla, Oaxaca, Morelos, Guerrero and Hidalgo, and is endemic to México. The flowers are hermaphroditic and self-incompatibles have nocturnal anthesis and are pollinated mainly by bats. This study addresses the effect of the orientation and the variation of floral traits associated with chiropterophyllous pollination syndrome on reproductive success (fruit-set and seed-set) of Pachycereus weberi in the TehuacánCuicatlán Biosphere Reserve. To determine the pattern of orientation, the type and number of reproductive structures in 42 individuals of $P$. weberi located at $\mathrm{Km} 73$ of the federal road Tehuacán-Oaxaca were recorded. Twelve automated devices were placed in three individuals of this species to record temperature and relative humidity. The variation in orientation, corolla diameter, mean and basal diameters, length and angle of inclination of the flowers and their relation to reproductive success was evaluated in 59 flowers of $P$. weberi in San José Chichihualtepec, Oaxaca. From the fruits and seed produced the fruit-set and seed-set, respectively, were estimated.

The number of ovules/ovary was estimated in a sample of 57 floral buds. The analyses indicate that $P$. weberi shows a pattern of southern orientation of its reproductive structures. This coincided with high temperatures during the day and higher values relative humidity during the night. This would benefit the development of flowers due to increased temperatures achieved by an equatorial orientation, so promoting meristematic activity. Moreover, the orientation and the mean diameter of the flower are the floral characters that explain the reproductive success of Pachycereus weberi in terms of production of fruits and seeds.

This suggests that flowers with larger mean diameter (a measure of size) and southern orientations are more successful in producing fruits and seeds. The experiment results suggest that the scent is an important character in reproductive success, although differences were not significant. So, the flowers orientation and the variability of floral characters have positive effects on pollination and post-pollination events. This study emphasizes the importance of the selection of floral traits exerted by bats and it's consequences on reproductive success in $P$. weberi. 


\section{INTRODUCCIÓN}

Las cactáceas columnares son un componente fisonómico dominante en diversas zonas áridas y semiáridas del continente americano. Su distribución se circunscribe a las regiones tropicales y subtropicales del continente, incluyendo como importantes reservorios de diversidad a Brasil, México y Perú (Gibson y Nobel, 1986; Sahley, 2001; Bustamante y Búrquez, 2005). En México, existen aproximadamente 70 especies de cactáceas columnares (Arias et al., 1997; Bravo-Hollis y SánchezMejorada, 1978), de las cuales 45 se encuentran en la vertiente del Pacífico Sur, región que comprende el Valle de Tehuacán-Cuicatlán y la depresión del Balsas (Bustamante y Búrquez, 2005). Esta región es considerada como una de las de mayor importancia por su diversidad de cactáceas columnares en el mundo (Valiente-Banuet et al., 1996; Casas et al., 1999; Bustamante y Búrquez, 2005). Entre las especies más representativas de esa región se cuentan Cephalocereus columna-trajani, Lemaireocereus hollianus, Neobuxbaumia tetetzo, N. macrocephala, N. mezcalaensis, Myrtillocactus geometrizans, M. schenckii, Stenocereus stellatus, S. pruinosus, S. griseus, Polaskia chichipe, P. chende y Pachycereus weberi, entre otras (Bravo-Hollis y Sánchez-Mejorada, 1978). Estas especies son de gran importancia ecológica porque proveen de recursos alimenticios indispensables a grandes poblaciones de murciélagos, aves e insectos que hacen uso del néctar, polen y frutos como alimento-(Fleming y Sosa, 1994; Valiente-Banuet et al., 1996; Nassar et al., 1997; Casas et al.,1999; Fleming et al., 2001; Arias-Cóyotl et al., 2006). 
Las cactáceas columnares, al igual que otros miembros de la familia, exhiben una gran variedad de hábitos y estructuras altamente especializadas que representan soluciones morfológicas, fisiológicas y ecológicas a las temperaturas extremas y a la baja disponibilidad de agua (Nobel, 1980; Gibson y Nobel, 1986; Zavala-Hurtado et al., 1998; Nobel y Loik, 1999; Altezor y Ezcurra, 2003; Valverde et al., 2007). Ejemplos de tales atributos son los tallos suculentos, la cubierta de espinas, la pubescencia apical y/o lateral, las costillas, los tubérculos, entre otros (Gibson y Nobel, 1986; Nobel y Loik, 1999). Pero también lo es la orientación no aleatoria en la que se encuentran dispuestas las estructuras reproductivas $\mathrm{y} / \mathrm{o}$ vegetativas tales como tallos, yemas, flores y frutos (e.g., Johnson, 1924; ZavalaHurtado et al., 1998; Tinoco-Ojanguren y Molina-Freaner, 2000; Aguilar de la Paz y Zavala-Hurtado, 2002).

Diversos estudios en cactáceas columnares reportan un acimut preferencial en la disposición de sus estructuras reproductivas orientadas hacia el ecuador, distribuyéndose con mayor frecuencia en las caras del tallo o ramas con mayor incidencia de radiación solar y temperaturas más elevadas (Gibson y Nobel, 1986; Nobel, 1986, 1988; Nobel y Loik, 1999; Tinoco-Ojanguren y Molina-Freaner, 2000). Se ha argumentado que la orientación preferencial influye de manera importante en la producción de frutos debido a que, por un lado, dicho patrón de distribución permite exponer a las yemas y flores a una temperatura adecuada para su desarrollo (Johnson, 1924; Zavala-Hurtado et al., 1998; Tinoco-Ojanguren y Molina-Freaner, 2000) y, por otro lado, permite optimizar la intercepción de Radiación 
Fotosintéticamente Activa (RFA), favoreciendo la captura de $\mathrm{CO}_{2}$ (Geller y Nobel, 1986; Tinoco-Ojanguren y Molina-Freaner, 2000).

El patrón de distribución preferencial de las estructuras reproductivas también ha sido interpretado como una adaptación que favorece un aumento considerable de la temperatura en los meristemos florales, promoviendo un incremento en la producción y desarrollo de botones, flores y frutos (Ehleringer et al., 1980; Yeaton et al., 1980; Geller y Nobel, 1986). De igual forma, la orientación de las flores podría tener efectos en la polinización y en los eventos post-polinización (Dafni, 1994; Patiño et al., 2002; Ushimaru et al., 2006). Aunque no existen reportes específicamente para cactáceas, algunos estudios han documentado que el aumento en la temperatura interna de las flores puede funcionar como recompensa para los visitantes florales (Hocking y Sharplin, 1965; Kudo, 1995; Patiño et al., 2002); y favorecer la fecundación, ya que las temperaturas ligeramente más elevadas aceleran los procesos de post-polinización, incluso pueden favorecer la maduración de frutos y semillas (Stanton y Galen, 1989; Totland, 1996). Sin embargo, es evidente que otros atributos florales como la forma, el color, tamaño y aroma; además de la orientación preferencial, son factores determinantes en el éxito de la producción de frutos y semillas (fruit-set y seed-set).

La forma, el tamaño y el color de las flores, probablemente son resultado evolutivo de la interacción entre las plantas y sus polinizadores (Feinsinger, 1983; von Helversen y von Helversen, 1999; Tschapka y Dressler, 2002), estos rasgos son 
considerados ornamentos sexuales que promueven el éxito reproductivo de las plantas (Moller y Eriksson, 1995). Rasgos florales cada vez más atractivos, atraen a más polinizadores lo cual incrementa el número de semillas y la eficiencia de la exportación de polen, siendo éstos componentes importantes de la adecuación de las plantas (Fleming y Sosa, 1994; Moller y Eriksson, 1995; Tschapka y Dressler, 2002).

Estudios realizados en distintas familias de angiospermas han revelado conjuntos de caracteres o síndromes de polinización (Faegri y van der Pijl, 1979; Fleming y Sosa, 1994; Nassar et al., 1997; Muchhala, 2007), los cuales son combinaciones particulares de colores, aromas, cantidades de recompensas, tamaños y formas asociados con grupos de polinizadores específicos (Pijl, 1961; Stebbins, 1970; Fenster et al., 2004; Raguso, 2004). Un número importante de cactáceas columnares presentan síndrome de polinización quiropterófilo (ValienteBanuet et al. 1996,1997).

El síndrome de polinización por quiropterofilia consiste en la atracción de murciélagos con ciertas características florales especificas, como son: antesis nocturna, flores grandes y robustas, grandes cantidades de polen y néctar, flores blancas con aromas intensos y numerosas anteras (Arita y Martínez del Río, 1990; Pijl, 1960; Howell, 1974; Faegri y van der Pijl, 1979; Grant y Grant, 1979; Fleming et al., 1993; Fleming y Sosa, 1994; Valiente-Banuet et al., 1996; Bustamante y Búrquez, 2005; Muchhala, 2007). Estas características han sido reportadas en especies de cactos columnares como Pachycereus pringlei (Moran, 1962; Fleming et al., 1993; 
Tinoco-Ojanguren y Molina-Freaner, 2000), Carnegiea gigantea (Johnson, 1924; Alcorn et al., 1962; Gibson y Nobel, 1986), entre otras, incluso la especie estudiada en la presenta investigación Pachycereus weberi (Valiente-Banuet et al., 1997).

El presente estudio aborda el efecto de la orientación de estructuras reproductivas y caracteres florales asociados al síndrome de polinización quiropterófilo sobre el éxito reproductivo (fruit-set: proporción de frutos exitosos y seed-set: proporción de semillas) de Pachycereus weberi en la región de TehuacánCuicatlán.

\section{ANTECEDENTES}

La orientación de yemas, flores y frutos, así como de meristemos, son características notables en varias especies de cactáceas, destacando las de forma de vida columnar y barriliforme. En uno de los primeros casos reportados en la literatura, Johnson (1924) analizó el caso del saguaro (Carnegiea gigantea). En dicho estudio el autor encontró que las yemas y flores se producen preferentemente en el lado sur de los tallos, coincidiendo con el registro de temperaturas óptimas altas, estimulando el crecimiento y maduración temprana de las flores.

Otros estudios, como el caso de Pachycereus pringlei en el hemisferio norte (Tinoco-Ojanguren y Molina-Freaner, 2000), y Trichocereus spp. en el hemisferio sur (Rundel, 1974; Nobel, 1981), reportan que el incremento en las temperaturas alcanzadas por la orientación ecuatorial mejora el desarrollo de las flores al favorecer la actividad meristemática. Ferocactus covillei y F. wislizenii en Arizona (Nobel, 1994) 
y Copiapoa spp. en Chile (Ehleringer et al., 1980) presentan orientación e inclinación del tallo hacia el ecuador, lo que les confiere claros beneficios en términos de la intercepción de luz solar. Gracias a este patrón de orientación, la temperatura de los tejidos de la regiones meristemática y florífera en el ápice del tallo, se incrementa durante el invierno y primavera favoreciendo el crecimiento y la floración (Ehleringer et al., 1980; Nobel, 1994).

Resumiendo, la distribución no aleatoria de estructuras reproductivas es un factor importante en el éxito de la producción de frutos y semillas, permitiendo exponer a los botones, flores y frutos a condiciones microambientales adecuadas para su desarrollo y crecimiento. Ushimaru et al. (2006), consideran que la orientación de las flores incluye dos dimensiones: a) ángulo de la flor (i.e. inclinada hacia arriba, inclinada hacia abajo, vertical u horizontal) y, b) dirección de la flor (i.e. norte, sur, este y oeste). Dichos componentes de la orientación podrían haber evolucionado por presiones selectivas del medio abiótico, como la temperatura y precipitación (Huang et al., 2002; Patiño et al., 2002; Galen y Stanton, 2003; Ushimaru y Hyodo, 2005; Ushimaru et al., 2006).

Algunos autores sugieren que la inclinación de las flores hacia "abajo" es una adaptación que evita el daño del polen y la dilución del néctar por la lluvia (Tadey y Aizen, 2001; Huang et al., 2002; Ushimaru y Hyodo, 2005); en cambio, la inclinación de las flores hacia "arriba" en dirección al sol en climas árticos y sub-árticos (heliotropismo), favorece el incremento de la temperatura interna de las flores, lo cual promueve la atracción de polinizadores, el crecimiento del tubo polínico y la 
maduración de frutos y semillas (Galen y Stanton, 2003). Este fenómeno también ocurre en flores tropicales de climas húmedos (Patiño et al., 2002).

Adicionalmente, la orientación de las flores es considerada un rasgo floral muy importante para atraer a polinizadores potenciales en algunas especies de angiospermas (Patiño et al., 2002; Ushimaru et al., 2006). De hecho, varios autores afirman que una ligera o drástica variación en alguna de estas características florales (orientación e inclinación), puede afectar la tasa de visita de polinizadores potenciales a las flores y, por consiguiente, afectar la producción de frutos y semillas (Totland, 1996; Patiño et al., 2002; Ushimaru y Hyodo, 2005).

Por otro lado, las flores de algunas cactáceas exhiben otros atributos especializados relacionados con su sistema de polinización y que también son determinantes en su éxito reproductivo.

Alrededor del $70 \%$ de las especies de cactáceas columnares presentan síndrome de polinización quiropterófilo (Valiente-Banuet et al., 1996; Nassar et al., 1997; Valiente-Banuet, 2002); es decir, rasgos florales y fenológicos que reflejan adaptaciones de las plantas a la polinización por murciélagos (van der Pijl, 1961; Faegri y van der Pijl, 1979; Knudsen y Tollsten, 1995). Este síndrome se presenta en cerca de 750 especies de 64 familias de angiospermas (Knudsen y Tollsten, 1995), pero en el caso particular de las cactáceas, este conjunto de adaptaciones incluyen las siguientes características florales (van der Pijl, 1961; Knudsen y Tollsten, 1995; Valiente-Banuet, 2002): 1) antesis nocturna, 2) flores grandes y robustas con corolas 
blancas, 3) gran producción de néctar y polen, 4) aromas desagradables y 5) ubicación particular de las flores que permiten el fácil acceso de los polinizadores.

A pesar de reconocer que los polinizadores han influido en la evolución de los caracteres florales (e.g., Galen, 1989; Medel et al., 2003), son pocos los estudios que han abordado cómo la variación de los caracteres florares influye en el éxito reproductivo en cactáceas.

\section{JUSTIFICACIÓN}

Existen en la literatura varios ejemplos de estudios que han abordado tanto la orientación preferencial de las flores como los síndromes de polinización en cactáceas (Johnson, 1924; van der Pijl, 1961; Moran, 1962; Faegri y van der Pijl, 1979; Ehleringer et al., 1980; Feinsinger, 1983; Geller y Nobel, 1986; Fleming et al., 1993; Nobel, 1994; Knudsen y Tollsten, 1995; Valiente-Banuet et al., 1996 y TinocoOjanguren y Molina-Freaner, 2000).

Sin embargo, sobre el patrón de orientación preferencial, éste ha sido documentado en cactos extratropicales (i.e., que habitan al norte del Trópico de Cáncer o al sur del Trópico de Capricornio), con pocos reportes sobre este fenómeno en especies intertropicales (Zavala-Hurtado et al. 1998; Valverde et al., 2006, 2007). En relación con los síndromes de polinización, no obstante que varios caracteres florales reflejan la adaptación de las plantas a la polinización por murciélagos (Knudsen y Tollsten, 1995; Muchhala, 2007), no existen reportes sobre el efecto de la 
variación de estos caracteres florales en el éxito reproductivo en especies de cactáceas.

El presente estudio aborda el caso de Pachycereus weberi, una cactácea arborescente gigante común en la parte sur de la Reserva de la Biosfera TehuacánCuicatlán. Además de su gran tamaño y abundantes ramas en forma de candelabro, $P$. weberi se caracteriza por la evidente orientación de sus estructuras reproductivas. No obstante, este fenómeno no ha sido estudiado en esta especie hasta la fecha. Por otro lado, aunque $P$. weberi es polinizada por murciélagos, presenta flores hermafroditas auto-incompatibles (Valiente-Banuet et al., 1997), no existen estudios que asocien la variación en la orientación y morfología de las flores con el éxito reproductivo en relación a la producción de frutos y semillas.

Por lo tanto, 1) ¿existe una producción asimétrica de flores de acuerdo con su orientación en las ramas? y 2) ¿varían con la orientación los atributos florales relacionados con la atracción de polinizadores?.

\section{HIPÓTESIS}

a) Si la distribución circular preferencial de las estructuras reproductivas en las ramas, fuera asimétrica, debido a que dicho patrón de distribución permite exponer a las yemas y flores a una temperatura adecuada para su desarrollo, se esperaría una mayor frecuencia de flores y frutos en las caras de las ramas con mayor incidencia de radiación solar y temperaturas más elevadas. 
b) $\mathrm{Si}$ los atributos florales más llamativos atraen más polinizadores incrementando la producción de frutos y semillas, se esperaría que la variación en la orientación de las flores y en los caracteres florales asociados con el síndrome de polinización quiropterófilo, se encuentren correlacionados con el éxito reproductivo en términos de proporción de frutos exitosos y proporción de semillas en $P$. weberi.

c) Si el aroma es uno de los caracteres fundamentales asociados con el síndrome de polinización quiropterófilo, esperamos probar experimentalmente que flores con aroma "aumentado" serán más exitosas en producir frutos y semillas que flores control.

\section{OBJETIVOS}

a) Determinar el patrón de distribución circular preferencial de las estructuras reproductivas (yemas, flores y frutos) y su relación con la variación diurna y nocturna de la temperatura y humedad relativa en diferentes ramas con distinta orientación en Pachycereus weberi.

b) Analizar el efecto de la orientación de las flores y la variación de los caracteres florales asociados al síndrome de quiropterofilia y su relación con el éxito reproductivo en términos de proporción de frutos exitosos y proporción de semillas en $P$. weberi. 
c) Evaluar el posible efecto del aroma como un carácter floral importante del síndrome de polinización quiropterófilo en el éxito reproductivo de $P$. weberi en términos de producción de frutos y semillas.

\section{MAteriales y métodos}

\subsection{Sistema de estudio}

Pachycereus weberi (J. M. Coulter) Backeb., es una planta arborescente de 10 a $15 \mathrm{~m}$ de alto (Figura 1). Presenta tallos con ramificación desde la parte media del tallo principal, con aréolas (2 a $2.5 \mathrm{~cm}$ de ancho) y espinas persistentes (1 a $11 \mathrm{~cm}$ de largo); las ramas son numerosas y verticales verde-glaucas (40 $\mathrm{cm}$ de ancho, aproximadamente); el número de costillas oscila entre 9 y 11; con un surco longitudinal interareolar.

Las flores miden de 7 a $11 \mathrm{~cm}$ de largo, son infundibuliformes, dispuestas cerca del ápice; el tubo receptacular mide de 6 a $8 \mathrm{~cm}$ de largo, con brácteas deltoides a triangulares, carnosas de 0.5 a $1 \mathrm{~cm}$ de largo; los tépalos internos son de 2 a $3 \mathrm{~cm}$ de largo; con estambres filamentosos entre 0.5 a $1 \mathrm{~cm}$ de largo, color blanco-amarillentos. Sus frutos miden entre 3.5 y $7 \mathrm{~cm}$ de largo y de 3 a $6 \mathrm{~cm}$ de ancho, con forma elipsoide, rojo-púrpura, semi-carnosos. Sus semillas son piriformes y miden de 2.5 a $3.5 \mathrm{~mm}$ de largo; a su vez, la testa presenta paredes celulares rectas, a veces sinuosas (Arias et al., 1997).

Las flores son hermafroditas, auto-incompatibles, presentan antesis nocturna y son polinizadas principalmente por murciélagos, aunque varios visitantes diurnos se 
observan en las flores al día siguiente (Valiente-Banuet et al., 1997). La época de floración de $P$. weberi ocurre desde finales de enero, hasta abril, mientras que la fructificación se concentra en los meses de marzo, abril y mayo (Córdova-Acosta et al., 2009). Las flores se localizan sub-apicalmente (laterales) en las ramas de esta planta arborescente.

Pachycereus weberi se distribuye en los estados de Morelos, Guerrero, Hidalgo, Puebla y Oaxaca. Se le conoce con los nombres comunes de "cardón", "chico", "órgano" y/o "candelabro". El tipo de vegetación dominada por esta especie de cactácea columnar se le denomina "cardonal" (Arias et al., 1997).

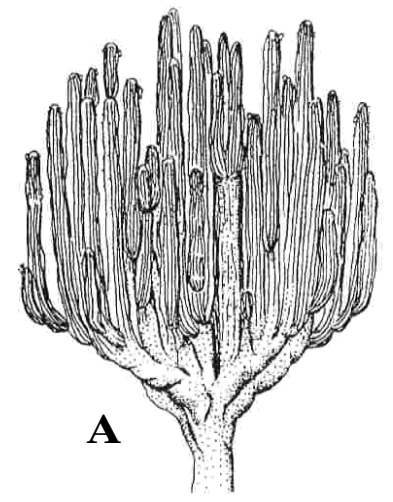

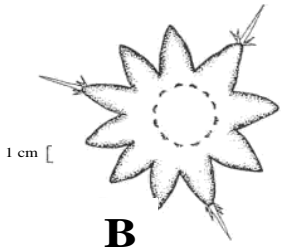

B

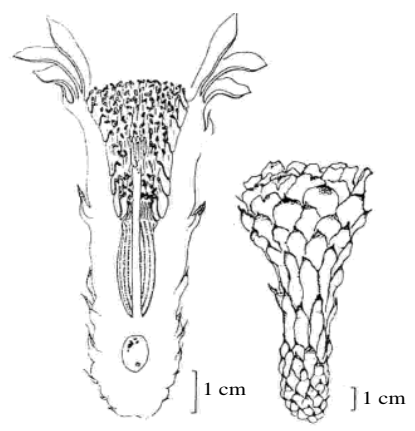

$\mathbf{E}$
$\mathbf{F}$

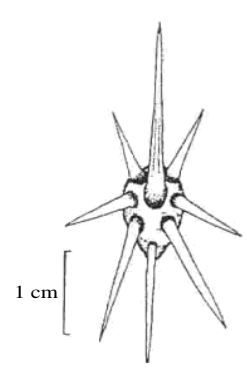

C
D

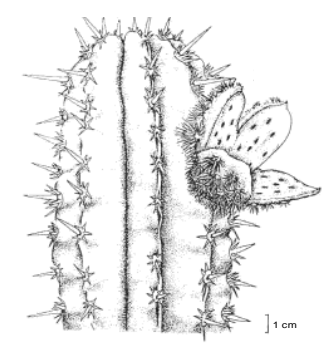

Figura 1. Pachycereus weberi. A) Forma de vida. B) Corte transversal de una rama. C) Aréola con espinas. D) Ápice de una rama con fruto. E) y F) Corte longitudinal de flor y parte externa (Modificado de Arias et al., 1997). 


\section{2 Área de estudio}

El presente trabajo se realizó en dos localidades de la Reserva de la Biosfera Tehuacán-Cuicatlán.

La primera localidad de estudio se localiza en el km 73 de la Carretera Federal 135 Tehuacán-Oaxaca, dentro de la Reserva de la Biosfera Tehuacán-Cuicatlán. Dicha localidad pertenece al Municipio de San José Miahuatlán, Puebla y sus coordenadas son $18^{\circ} 15^{\prime} 32^{\prime \prime} \mathrm{N}$ y $97^{\circ} 19^{\prime} 22^{\prime \prime}$ W con una altitud de $1550 \mathrm{~m}$ s.n.m. (Figura 2).

El clima en esta región es semiárido con lluvias en verano. La temperatura promedio anual es de $20^{\circ} \mathrm{C}$ y la precipitación promedio anual es de $400 \mathrm{~mm}$ (ZavalaHurtado et al., 1998). El tipo de vegetación dominante en la región es matorral xerófilo (Rzedowski, 1978).

El segundo sitio de estudio se ubicó en San José Chichihualtepec, Municipio de Santiago Chazumba, Oaxaca. El sitio se localiza en las coordenadas $18^{\circ} 11^{\prime} 35^{\prime \prime} \mathrm{N}$ y $97^{\circ} 48^{\prime} 20^{\prime \prime}$ W con una altitud de 1589 m.s.n.m. (ver Figura 2).

La temperatura media anual es de $20.5^{\circ} \mathrm{C}$ y la precipitación promedio anual es de $680 \mathrm{~mm}$. El tipo de vegetación según Rzedowski (1978) es bosque tropical caducifolio y el clima en la mayor parte del año es templado seco, con lluvias en el verano. 


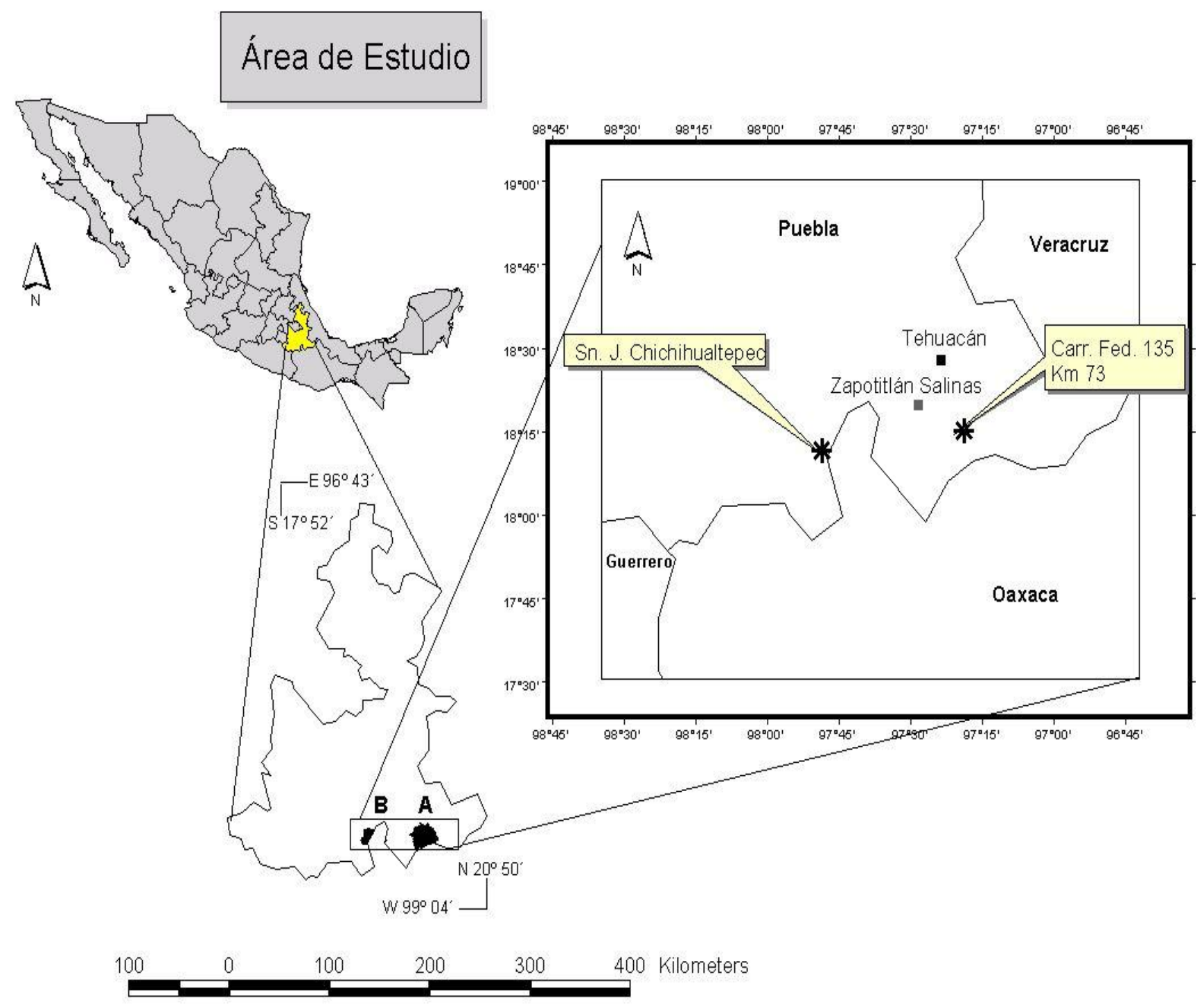

Figura 2. Mapa de las dos localidades de estudio: A) Carretera Federal 135, Tehuacán-Oaxaca, Km 73, pertenece al Municipio de San José Miahuatlán, Puebla. B) San José Chichihualtepec, Oaxaca, Municipio de Santiago Chazumba, Oaxaca. Ambas localidades se encuentran ubicadas en la Reserva de la Biosfera de Tehuacán-Cuicatlán, México. 


\subsection{Determinación de la orientación preferencial de estructuras reproductivas}

La evaluación de la orientación preferencial de estructuras reproductivas en $P$. weberi se realizó en el primer sitio de estudio en la época de floración durante febrero de 2007 y de 2008.

El área de cobertura de 42 individuos se dividió en cuatro sectores (Figura 3). Para cada individuo se eligió al azar uno de los cuatro sectores y, en el sector seleccionado, se escogieron las tres ramas más altas. Lo anterior se hizo para evitar, en lo posible, el efecto de la obstrucción de ramas contiguas sobre la orientación de estructuras reproductivas. En cada rama se seleccionó una estructura reproductiva específica (yema, flor y/o fruto) como referencia, y se registró la orientación de la costilla en la cual se ubicó dicha estructura utilizando una brújula Brunton.

A partir de la costilla de referencia (i.e., costilla 1, Figura 4) se contabilizó el número total de costillas en las ramas, el tipo de estructura reproductiva encontrada en cada costilla (yema, flor y/o fruto) y el número total de las mismas. El conteo se realizó siguiendo la dirección de las manecillas del reloj (Figura 4). Posteriormente, a partir del acimut de referencia (i.e., costilla 1; Figura 4), se estimó el acimut de cada una de las costillas dividiendo $360^{\circ}$ entre el número total de costillas de la rama (entre 9 y 11 costillas; Arias et al., 1997). De este modo, a cada estructura reproductiva se le asignó la orientación de la costilla en la que se encontraba presente. Estos cálculos se realizaron en las tres ramas seleccionadas de los 42 individuos de $P$. weberi. 
El análisis de la orientación de estructuras reproductivas se evaluó por medio de estadística circular (Zar, 1999) utilizando el programa ORIANA v 2.02 (Kovach, 2004).

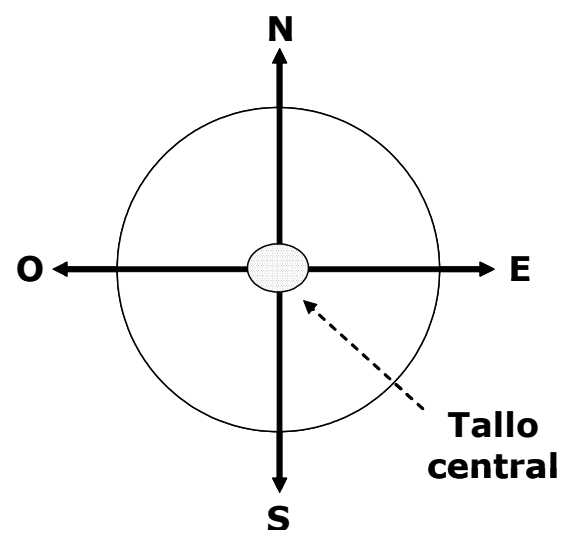

Figura 3. Área de cobertura de cada individuo dividida en cuatro sectores.

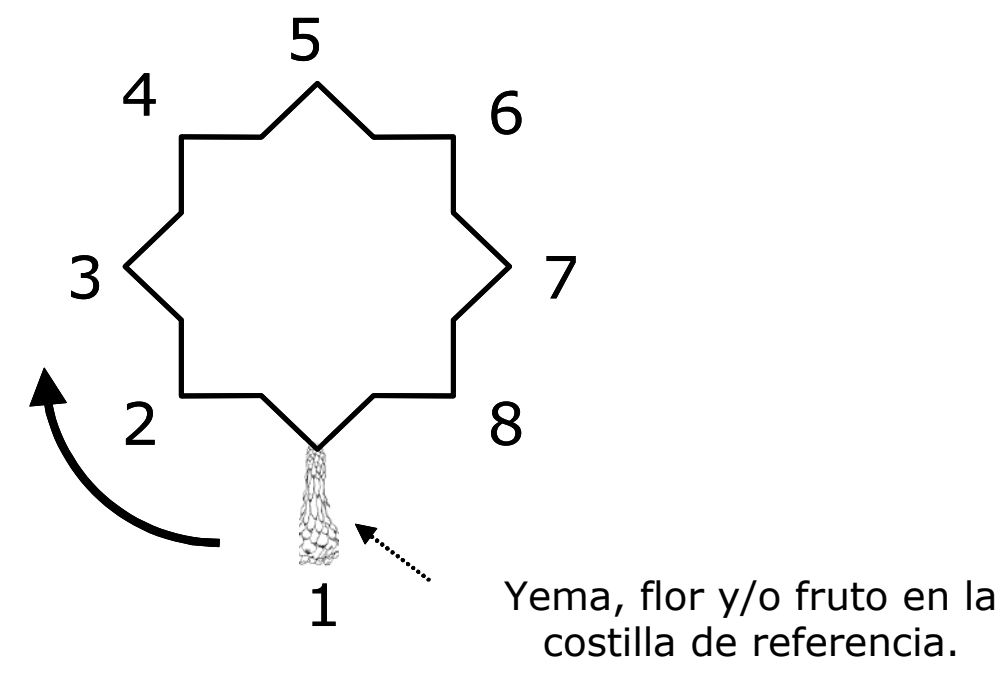

Figura 4. Conteo del número de costillas y estructuras reproductivas de acuerdo al sentido de las manecillas del reloj, comenzando con la flor de la costilla de referencia (i.e., costilla 1). 


\subsubsection{Registro de las condiciones microambientales: temperatura y humedad relativa}

Para registrar la temperatura y humedad relativa en torno a las estructuras reproductivas de $P$. weberi, en el primer sitio de estudio se emplearon 12 dispositivos automatizados de registro de datos ambientales, conocidos como $\mathrm{HOBOs}(\mathrm{HOBO} \cap$ Modelo H08-004-02, Onset Computer Corporation, Estados Unidos). Los dispositivos fueron colocados en tres ramas de tres individuos distintos (i.e., una rama en cada individuo), seleccionados aleatoriamente.

En la parte superior de cada rama se colocaron cuatro dispositivos orientados hacia cada uno de los puntos cardinales (norte, sur, este y oeste) (Figura 5).

Los dispositivos fueron programados con anterioridad a su colocación para realizar lecturas de temperatura y humedad relativa en intervalos de cinco minutos por 24 horas. 


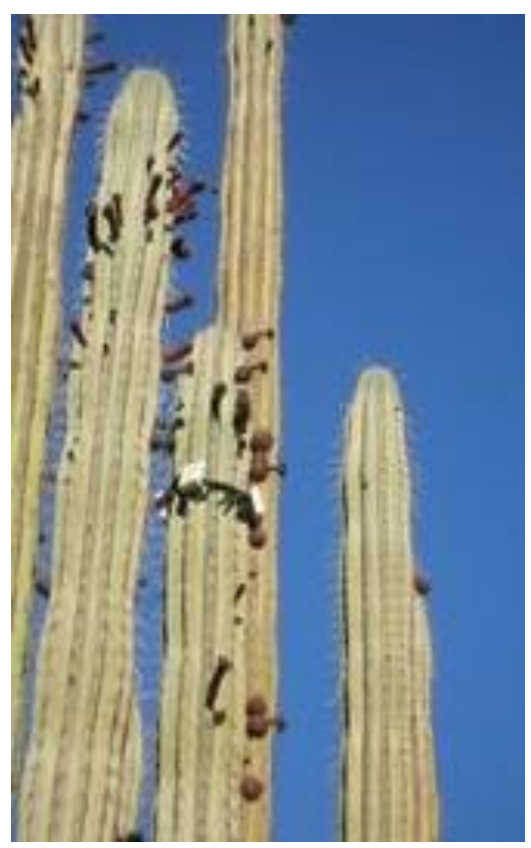

Figura 5. HOBOs colocados en la parte superior de una rama. También se puede apreciar algunas yemas o botones florales, flores y frutos de Pachycereus weberi.

\subsection{Medición de caracteres florales y su relación con el éxito reproductivo}

En la localidad de San José Chichihualtepec, Oaxaca, se llevó a cabo un estudio para determinar la variación de los caracteres florales asociados al síndrome de polinización por murciélagos y su posible relación con el éxito reproductivo en $P$. weberi. El estudio se realizó durante la época de floración de $P$. weberi entre los meses de febrero, marzo y abril por dos años consecutivos (2009 y 2010).

En función de su disponibilidad, 59 flores recién abiertas de 14 individuos fueron seleccionadas (37 flores en 2009 y 22 en 2010). Aunque otras flores estaban disponibles, su acceso para realizar mediciones era físicamente imposible. 
Cada flor fue etiquetada con rectángulos de madera de $4 \times 2 \mathrm{~cm}$, sujetos a la base de cada flor con un alambre (Figura 6), y en las cuales se midieron, in situ, los siguientes caracteres florales utilizando un vernier digital (Mitutoyo, Japón): longitud, diámetro de la corola (perianto), diámetro medio y basal (Figura 7).

El ángulo de inclinación de la flor con respecto a la vertical de la rama y su orientación se midieron utilizando un inclinómetro (Figura 8) y una brújula Brunton, respectivamente. También se registraron dos variables adicionales que reflejan el contexto en el que se producen las flores: la altura de cada una de las flores etiquetadas con respecto a la planta, empleando un hipsómetro digital (Vertex Haglöf $A B$, Suecia) y la densidad de estructuras, contabilizando la abundancia de estructuras reproductivas (i.e. botones, flores y/o frutos) en la ramificación dónde se encontraba la flor focal (i.e., flor etiquetada).

Después de las mediciones, las flores fueron encapuchadas con bolsas de tul blanco de $15 \times 15 \mathrm{~cm}$ sujetadas a la base de la flor con hilo de cáñamo (Figura 9). Esto se realizó con el fin de garantizar la recuperación de los frutos producidos.

Después de 30 días aproximadamente, los frutos producidos por las flores etiquetadas (i.e., la proporción de frutos exitosos) fueron colectados (Figura 10) y colocados en bolsas de papel, para posteriormente contabilizar y pesar en el laboratorio el número de semillas producidas. Las semillas fueron depositadas en bolsas de papel y almacenadas en el laboratorio. 

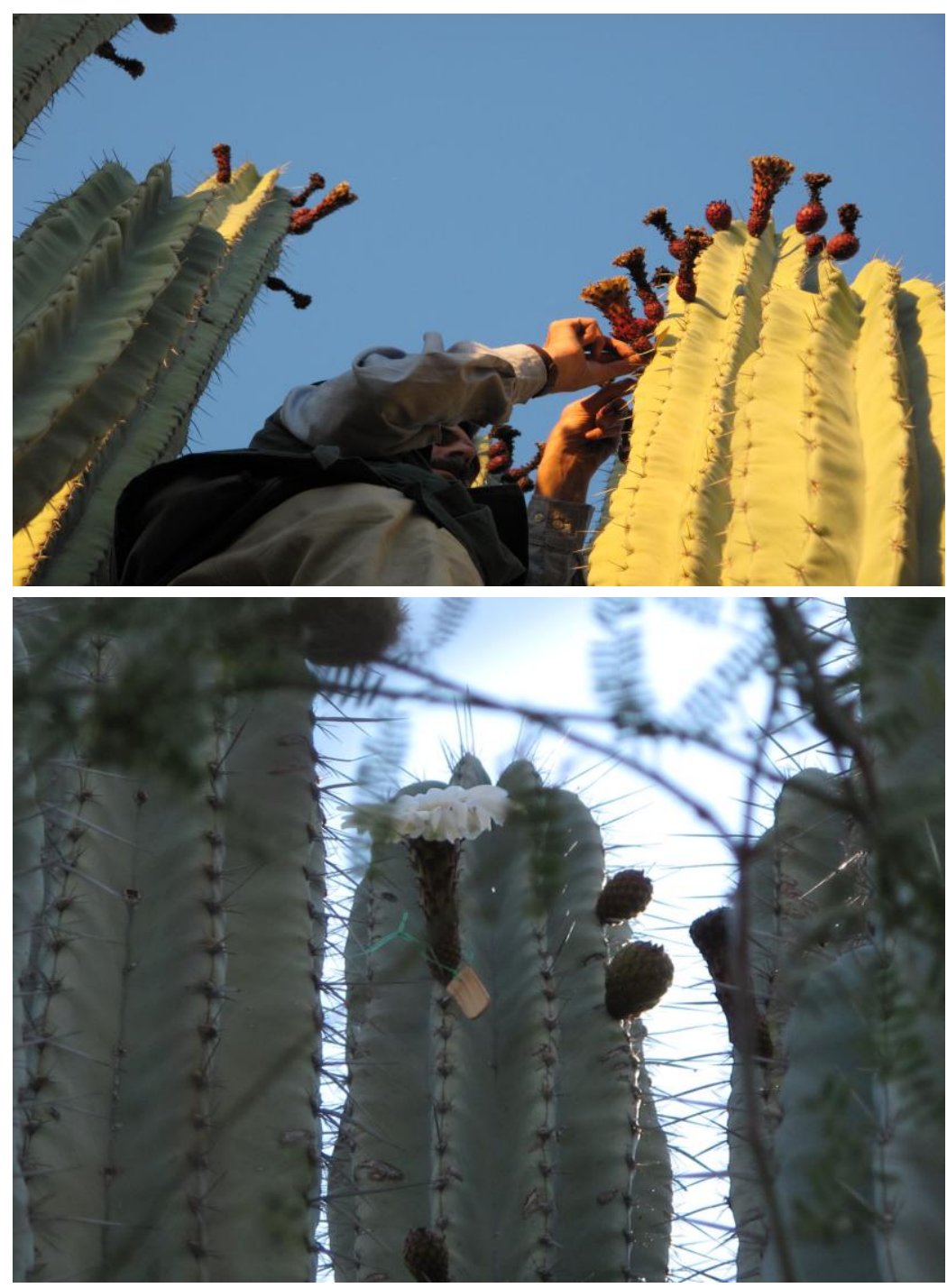

Figura 6. Flores medidas in situ y etiquetadas de Pachycereus weberi en San José Chichihualtepec, Oaxaca. 


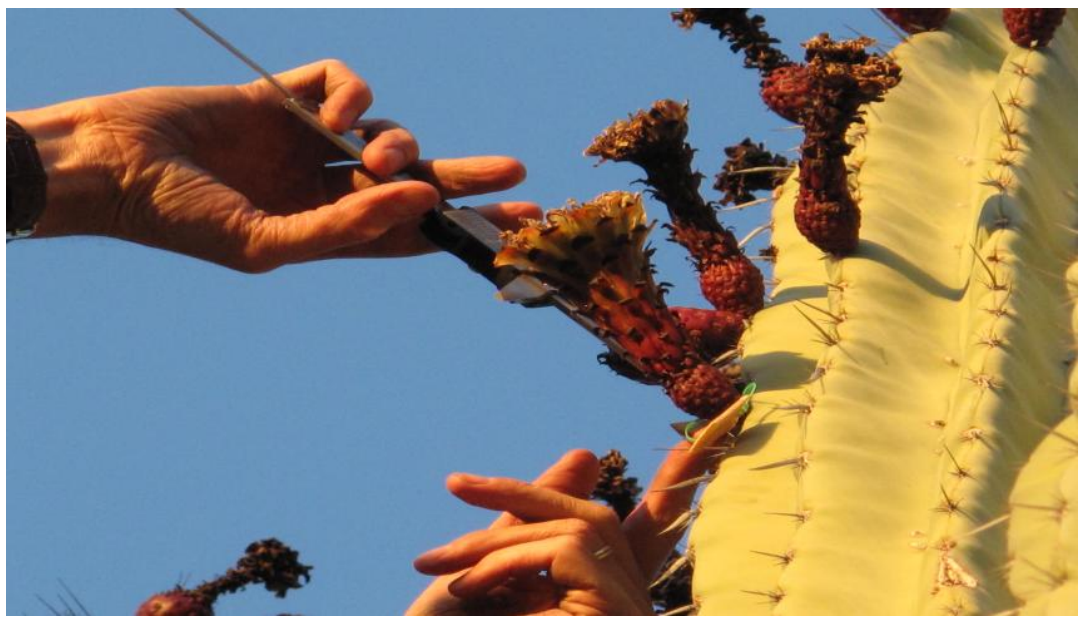

Figura 7. Medición de la longitud de una de las flores en un individuo de $P$. weberi en San José Chichihualtepec, Oaxaca.

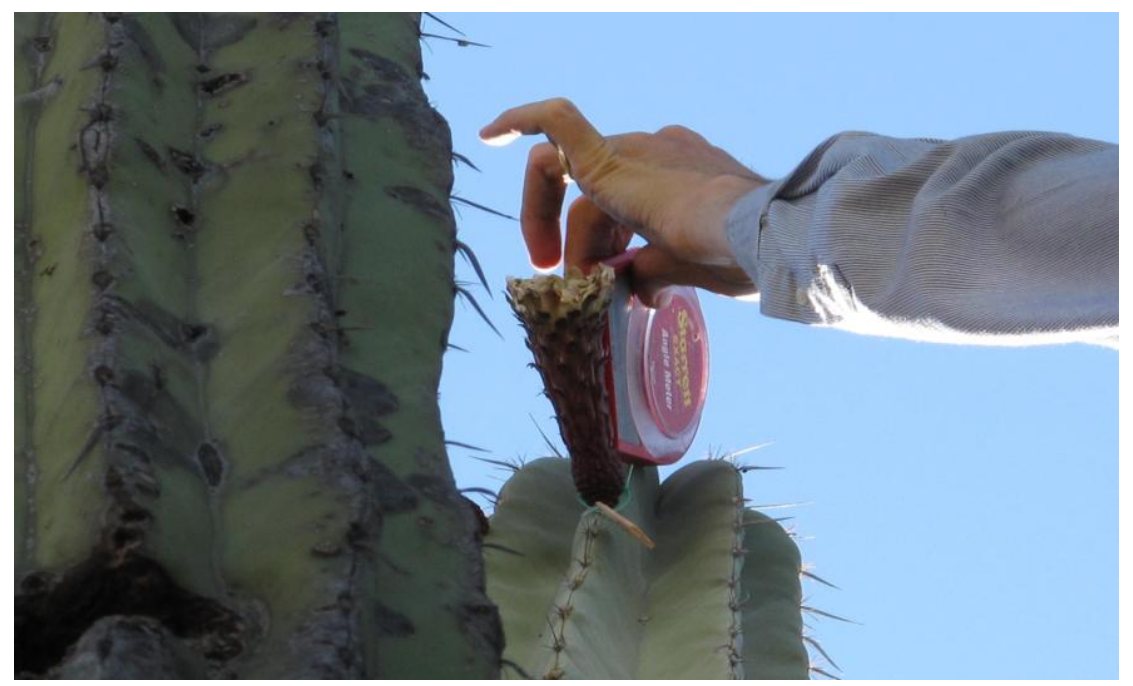

Figura 8. Medición in situ del ángulo de inclinación en una flor con respecto a la vertical de la rama en un individuo de $P$. weberi en San José Chichihualtepec, Oaxaca. 


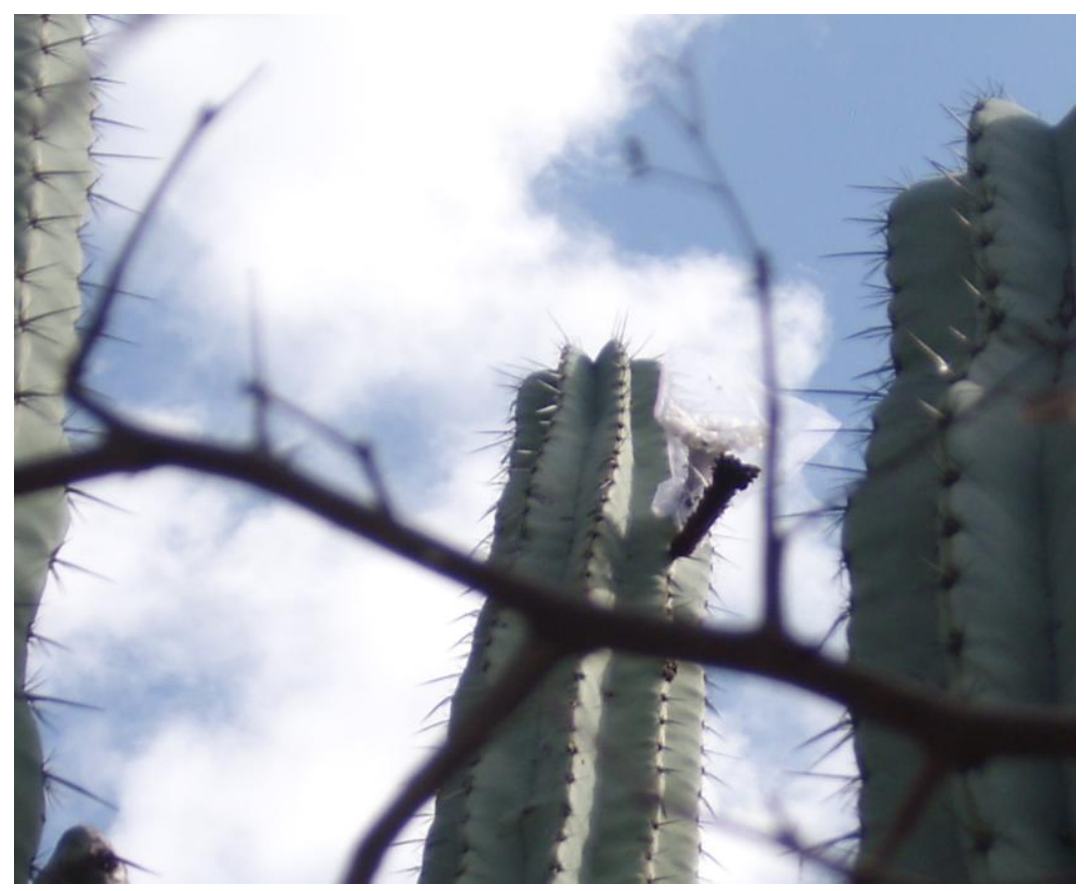

Figura 9. Flor medida in situ, etiquetada y embolsada para garantizar la recuperación del posible fruto producido, en uno de los individuos de $P$. weberi en San José Chichihualtepec, Oaxaca.

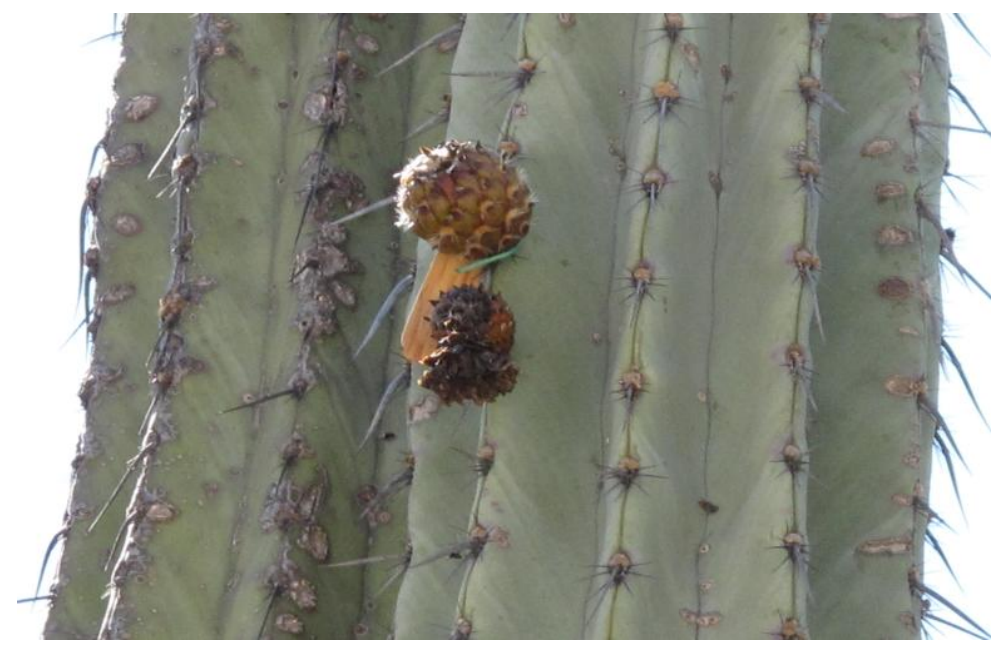

Figura 10. Fruto producido por una de las flores medidas in situ en uno de los individuos de $P$. weberi en San José Chichihualtepec, Oaxaca. 
Para estimar el número de óvulos por ovario, necesarios para calcular la proporción de semillas producidas (número de semillas por fruto/ número de óvulos por ovario), se realizó una colecta de 57 yemas florales en 18 individuos de $P$. weberi, incluidos los 14 individuos con flores marcadas mencionados anteriormente. La orientación de cada una de ellas fue registrada con una brújula Brunton. Las yemas colectadas se almacenaron en bolsas de plástico con una solución fijadora de tejidos (F.A.A., Formaldehído-Alcohol etílico-Ácido acético) y se etiquetaron con papel albanene. En el laboratorio se procedió a contar el número de óvulos por ovario en cada una de las yemas colectadas. Utilizando un bisturí, los ovarios fueron diseccionados en cuatro partes, procurando que cada uno de los cortes fuera simétrico con respecto a los demás. Se eligió uno de ellos al azar y se colocó en una caja de Petri con un poco de agua destilada en un microscopio estereoscópico para facilitar su conteo.

Se utilizó un alfiler entomológico para separar con mucho cuidado cada uno de los óvulos, formando pequeños grupos de ellos dentro de la caja de Petri, los cuales se contabilizaron empleando un contador de mano. El número de óvulos obtenido en ese cuarto de ovario fue multiplicado por el número restante de los cortes realizados en ese ovario, para así obtener una estimación del número total de óvulos por ovario. Este procedimiento se realizó en toda la colecta.

Debido a que el número de yemas colectadas varió entre individuos (de 1 a 6 yemas, aproximadamente), un valor único o promedio del número de óvulos por individuo fue utilizado para estimar la proporción de semillas producidas. 
Para cada uno de los frutos exitosos, derivados de las flores etiquetadas, la proporción de semillas producidas se calculó dividiendo el número de semillas entre el valor único o número promedio de óvulos correspondiente al individuo.

Para medir el grado de asociación entre los caracteres florales medidos in situ, la altura de las flores con respecto a la planta y la densidad de estructuras se utilizaron pruebas de correlación de Pearson (Zar, 1999) utilizando el programa estadístico XLStat v. 2010 (Kovach, 2010) o pruebas de correlación circular-lineal empleando el programa de estadística circular ORIANA v. 2.02 (Kovach, 2004), según fuera el caso.

Por otro lado, se analizó el grado de asociación entre la orientación de las yemas florales colectadas y el número promedio de óvulos por ovario utilizando una prueba de correlación circular-lineal (Zar, 1999). Los análisis se realizaron empleando el programa estadístico XLStat v. 2010 (Kovach, 2010). Los análisis de estadística circular se efectuaron mediante el programa ORIANA v. 2.02 (Kovach, 2004).

El éxito o fracaso en la producción de frutos derivados de las flores etiquetadas se registró como una variable binaria, es decir, 1 o 0 , respectivamente (proporción de frutos exitosos).

Para probar si la variación en los caracteres florales medidos in situ, además de la altura y densidad de estructuras reproductivas tenía un efecto en la proporción de frutos exitosos, se utilizó un análisis de regresión logística múltiple (Neter et al., 
1996; Mendenhall y Sincich, 2003). Este análisis se realizó empleando el programa estadístico XLStat v. 2010 (Kovach, 2010).

Para probar si la variación en los caracteres florales medidos in situ, la altura y densidad de estructuras tenía un efecto sobre el número de semillas por fruto y la proporción de semillas, se utilizaron análisis de regresión múltiple (Zar, 1999) empleando el programa JMP (SAS, 1999).

Los anteriores análisis permitieron explorar las posibles relaciones de los caracteres florales, altura de la flor y densidad de estructuras con el éxito reproductivo, en términos de la proporción de frutos exitosos, número de semillas por fruto o proporción de semillas producidas en $P$. weberi.

\subsubsection{Experimento para evaluar el efecto del aroma como carácter floral y su relación con el éxito reproductivo}

Se realizó un experimento tomando en cuenta al aroma como un carácter floral que atrae a los polinizadores (murciélagos). Dicho experimento consistió en "incrementar el aroma" de las flores naturales de P. weberi (Raguso, 2006).

Para llevar a cabo esto, se colectaron dos flores recién cortadas de un individuo de $P$. weberi y se trituraron en un mortero de porcelana al cual se le añadieron $3 \mathrm{ml}$ de aceite mineral. Posteriormente, se obtuvo el extracto de las flores que fue colocado dentro de tubos eppendorf de $2 \mathrm{ml}$ hasta llenarlos.

En diez individuos de $P$. weberi se eligieron dos botones florales "a punto de abrir", localizados en ramas distintas, aunque cercanas y con orientación similar en la 
medida de lo posible. Se colocaron dos tubos eppendorf con el extracto de las flores (tratamiento con aroma "incrementado") en la base de uno de los botones florales por medio de un alambre fino, de tal forma que quedarán sujetados sin caerse al suelo (Figura 11).

De la misma forma, en el otro botón floral se colocaron dos tubos eppendorf sin extracto (tratamiento control) (Figura 12).

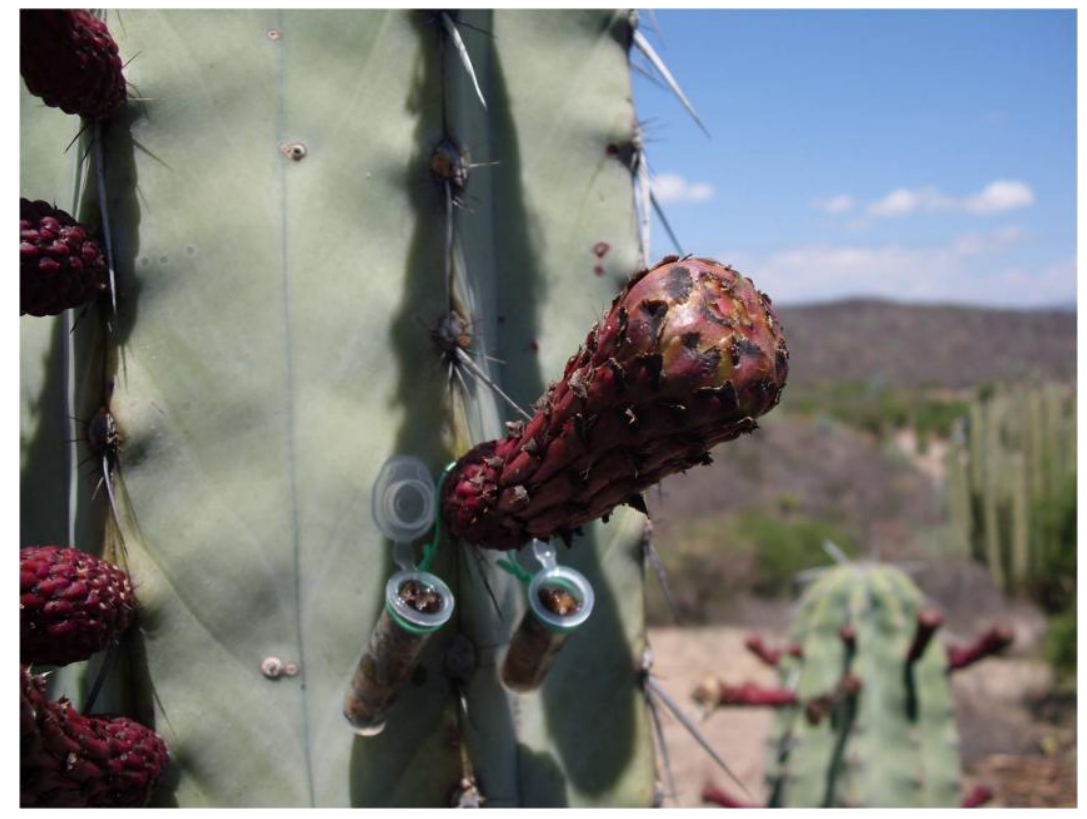

Figura 11. Tratamiento experimental con el "aroma aumentado" en un botón floral "a punto de abrir" en un individuo de $P$. weberi en San José Chichihualtepec, Oaxaca. 


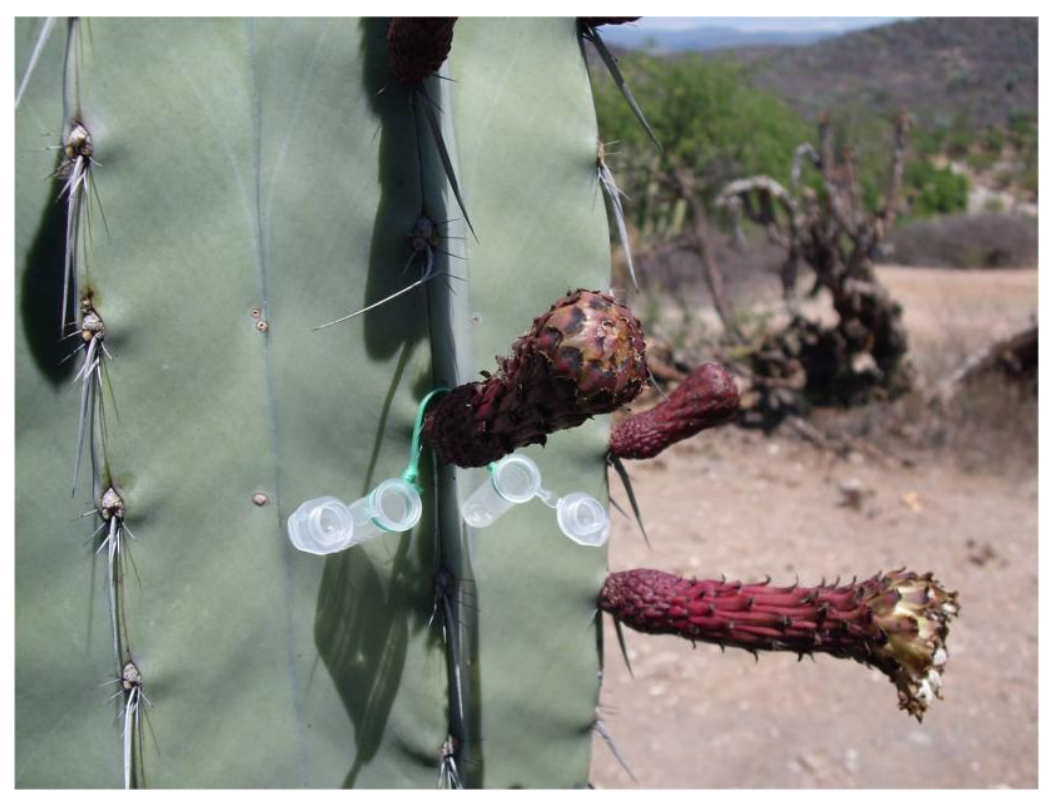

Figura 12. Tratamiento control en un botón floral "a punto de abrir" en un individuo de $P$. weberi en San José Chichihualtepec, Oaxaca.

Después de 30 días se colectaron los frutos obtenidos en ambos tratamientos y se contabilizó en el laboratorio el número de semillas producidas en cada fruto. La proporción de frutos exitosos fue evaluada con una prueba de $\chi^{2}$ pareada (Rufibach, 2011). El número de semillas por fruto y la proporción de semillas producidas fueron evaluados con pruebas de $t$-pareadas (Zar, 1999) empleando el programa estadístico NCSS (Hintze, 2001). 


\section{RESULTADOS}

\subsection{Orientación de estructuras reproductivas}

Los análisis estadísticos circulares muestran que la orientación de las estructuras reproductivas (i.e., yemas, flores y frutos) de $P$. weberi presentan un patrón preferencial sureño en 2007 y 2008. De hecho, no se detectaron diferencias significativas entre ambos años ( $F$ de Watson-Williams $=0.003, p=0.956)$. La dirección media (i.e., media circular, Zar 1999) fue significativa y corresponde con un acimut Sur (media \pm error estándar; $175.28^{\circ} \pm 4.12^{\circ}$, Figura 13 y Tabla 1 ). Sin embargo, aunque la orientación preferencial común es el sur, existen diferencias significativas entre las distintas estructuras reproductivas. En este sentido, la orientación media de las yemas difiere significativamente de aquella de flores ( $F=$ 14.3, $p<0.0001)$ y frutos $(F=34.5, p<0.0001$; Figura 14$)$, mientras que entre flores $\mathrm{y}$ frutos no existen diferencias $(F=0.009, p=0.924)$.

De acuerdo con lo anterior, las estructuras reproductivas presentan una distribución sureña, sin embargo las yemas se distribuyen hacia el suroeste $\left(229.43^{\circ}\right.$ $\pm 14.52^{\circ}$, Figura $14 \mathrm{~A}$ ), mientras que la distribución de flores y frutos es prácticamente $\operatorname{sur}\left(169.46^{\circ} \pm 12.69^{\circ}\right.$ y $168.29^{\circ} \pm 4.04^{\circ}$, respectivamente; Figura $14 \mathrm{~B}$ y C; Tabla 1$)$. 
Tabla 1. Estadísticos circulares básicos de la orientación de estructuras reproductivas de Pachycereus weberi (2007-2008).

\begin{tabular}{lcccc}
\hline & $\begin{array}{c}\text { Estructuras } \\
\text { reproductivas }\end{array}$ & Yemas & Flores & Frutos \\
\hline Tamaño de muestra $(n)$ & 592 & 131 & 41 & 420 \\
Dirección media $(\mu)$ & $175.28^{\circ}$ & $229.43^{\circ}$ & $169.46^{\circ}$ & $168.29^{\circ}$ \\
Concentración & 0.842 & 0.495 & 1.059 & 1.037 \\
$\mathrm{DE}_{\text {circular }}$ & $78.84^{\circ}$ & $96.77^{\circ}$ & $70.53^{\circ}$ & $71.30^{\circ}$ \\
EE $_{\text {circular }}$ & 4.12 & $14.52^{\circ}$ & $12.69^{\circ}$ & $4.04^{\circ}$ \\
Intervalo de Confianza $95 \%$ & $167.2^{\circ}-183.4^{\circ}$ & $201.0^{\circ}-257.9^{\circ}$ & $144.6^{\circ}-194.4^{\circ}$ & $160.4^{\circ}-176.2^{\circ}$ \\
Prueba de uniformidad de & 89.13 & 7.5 & 9.0 & 89.2 \\
Rayleigh $Z(p)$ & $(<0.0001)$ & $(0.00052)$ & $(7.89 \mathrm{E}-5)$ & $(<0.0001)$ \\
\hline
\end{tabular}

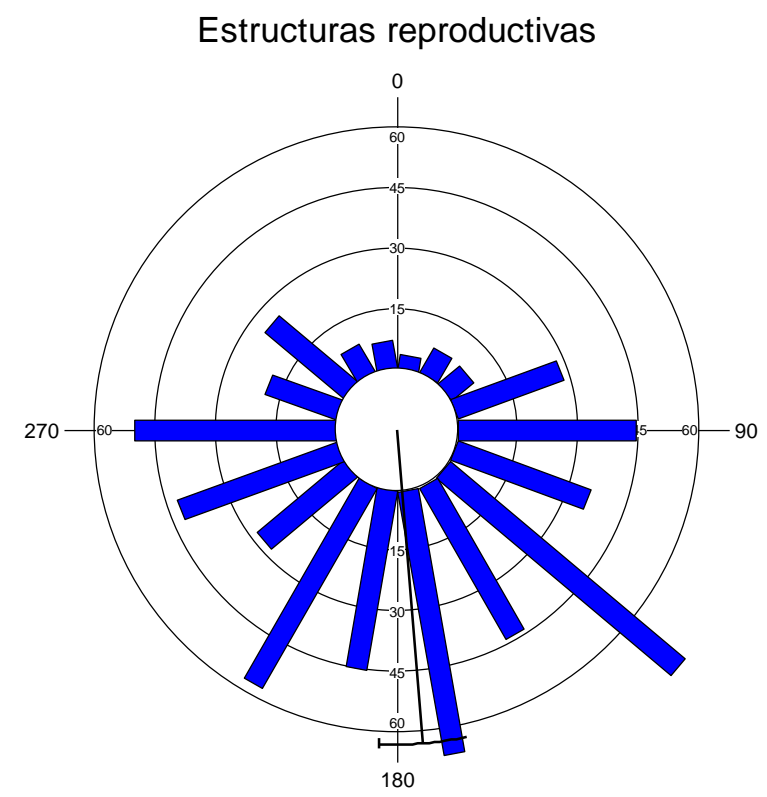

Figura 13. Distribución de frecuencia circular de estructuras reproductivas de Pachycereus weberi (2007-2008). 

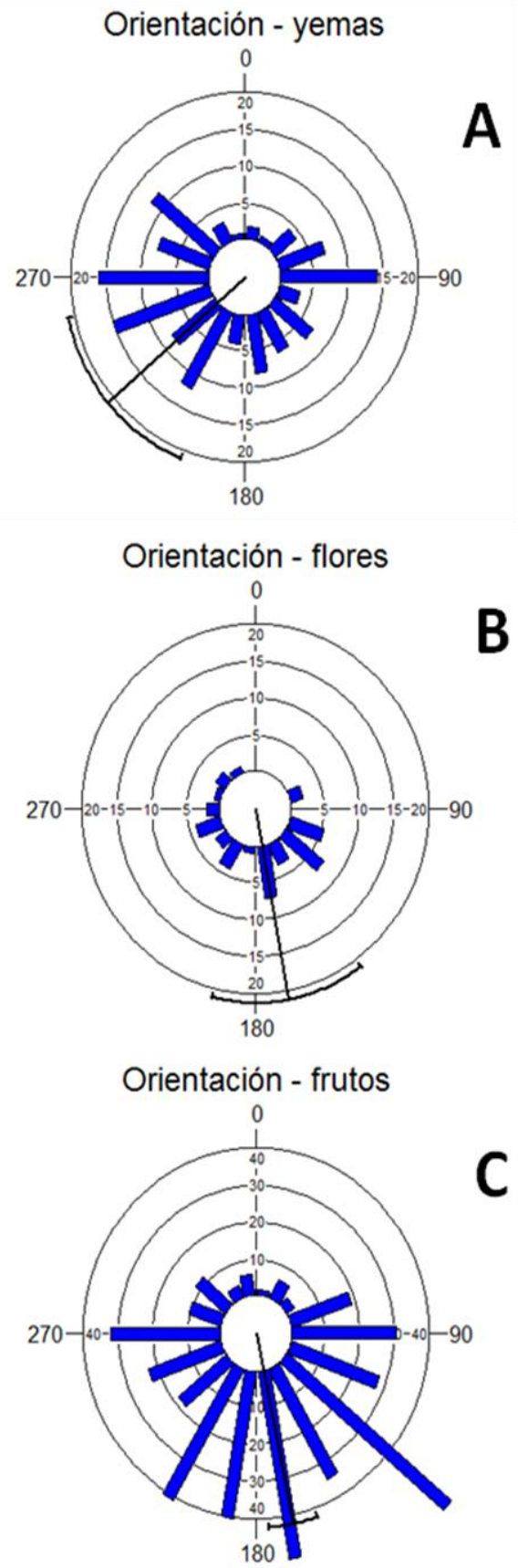

Figura 14. Distribución de la frecuencia circular de A) yemas florales, B) flores y C) frutos de Pachycereus weberi (2007-2008). 


\subsubsection{Registro de las condiciones microambientales: temperatura y humedad relativa}

Con respecto al registro de las condiciones microambientales, las marchas de temperatura no muestran diferencias apreciables durante la noche entre las cuatro direcciones (norte, sur, este y oeste), pero si durante el día, cuando el lado sur presenta la temperatura promedio más alta (Figura 15A). Los registros de humedad relativa, por el contrario, muestran diferencias sólo durante la noche, cuando el lado sur presenta la humedad relativa más alta (Figura 15B).

De acuerdo con lo anterior, la dirección hacia dónde se concentra el mayor número de estructuras reproductivas concordó con aquella de las temperaturas más altas durante el día y la humedad relativa más alta durante la noche. 


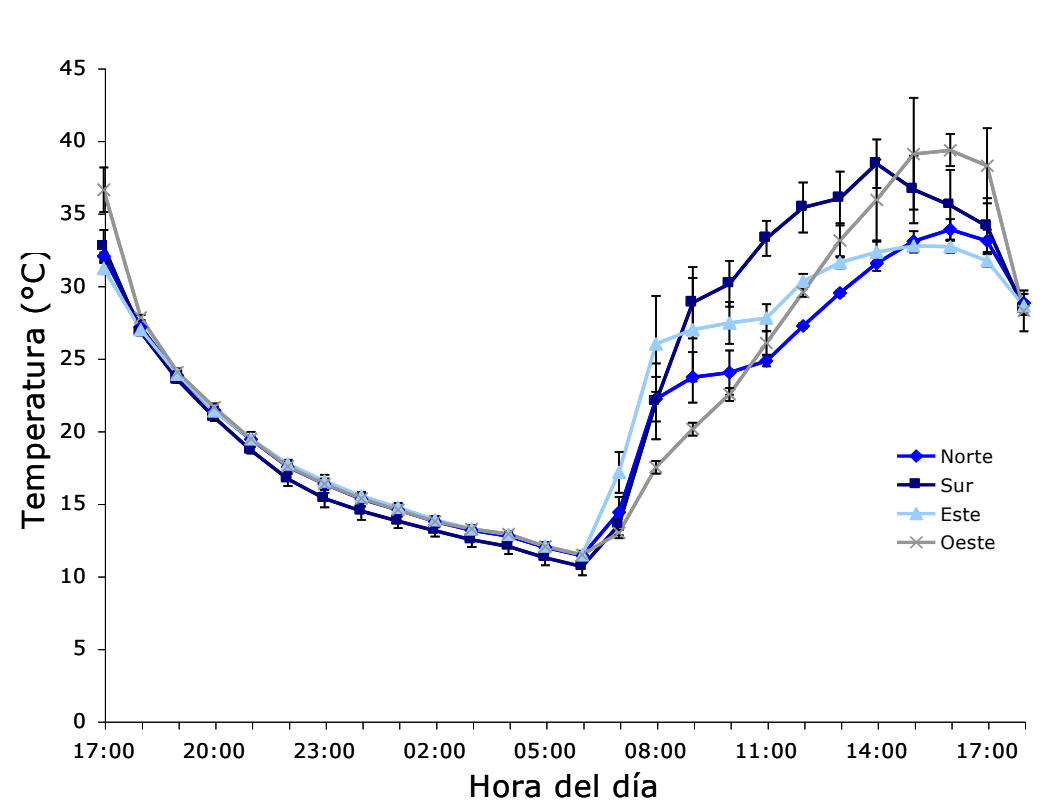

A

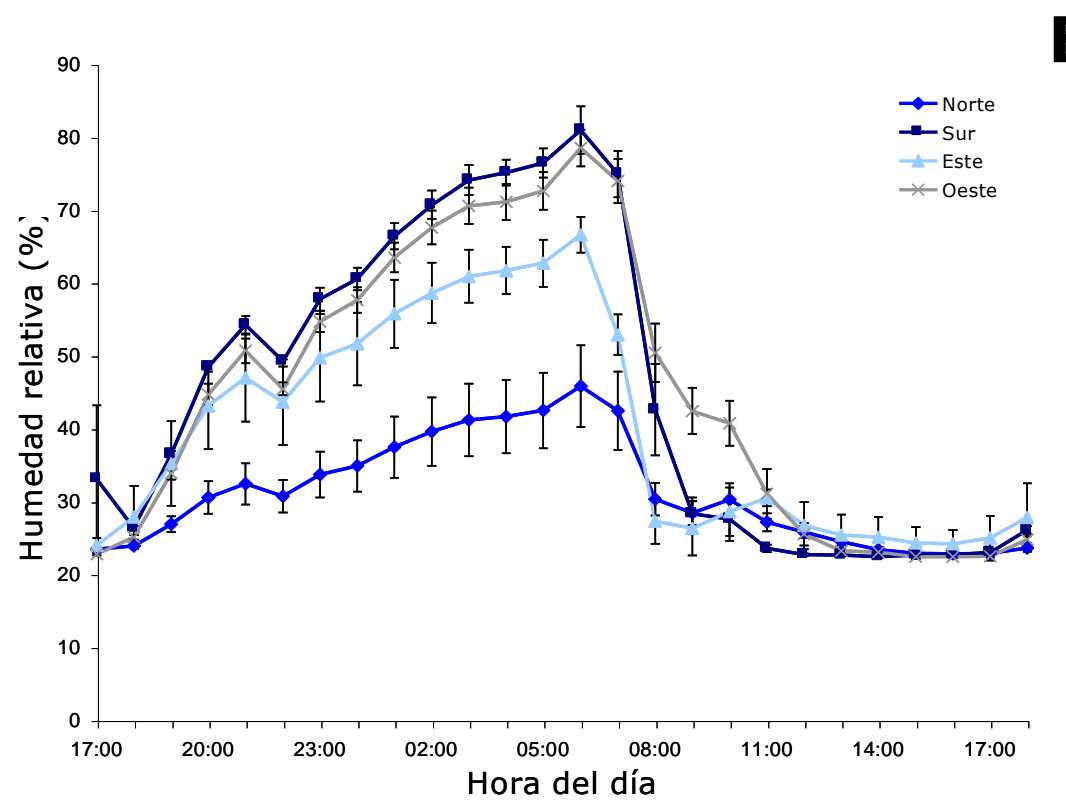

Figura 15. Marchas de temperatura (A) y humedad relativa $(B)$ en los cuatro puntos cardinales. Los datos son promedios de tres ramas de tres individuos distintos de Pachycereus weberi. Las barras representan $\pm \mathrm{EE}$. 


\subsection{Variación de los caracteres florales y su relación con el éxito reproductivo}

Del total de flores etiquetadas en ambos años $(n=59)$, se produjeron 24 frutos de los cuales 13 correspondieron a 2009 y 11 a 2010. Debido al modesto tamaño de muestra, todos los análisis subsecuentes fueron realizados con los datos de ambos años en conjunto.

Debido a que la orientación de las 59 flores etiquetadas varía de $29^{\circ}$ a $177^{\circ}$, no fue necesario el uso de la estadística circular. En este sentido, cabe resaltar que la única correlación significativa de la orientación con algún otro carácter floral fue con el diámetro de la corola $(r=0.2704, p=0.0383)$. Esto sugiere que las flores con corolas más grandes se asocian con orientaciones sur-sureste (Figura 16).

Con respecto al resto de los caracteres florales, se detectó que el diámetro de la base de las flores está correlacionado positivamente con la longitud $(r=0.5937, p$ $<0.0001)$ y el diámetro medio $(r=0.4054, p=0.0014)$, mientras la inclinación de la flor se correlaciona negativamente con la altura de la flor con respecto a la planta $(r=$ -0.4582, $p=0.0003$ ). Esto último sugiere que las flores tienden a ser más verticales cuando se producen en las regiones más apicales de las ramas.

También se detectó que ciertos caracteres florales son afectados negativamente por un incremento en la densidad de estructuras, tal es el caso de la longitud $(r=-0.3186, p=0.0139)$, diámetro de la base $(r=-0.3520, p=0.0063) \mathrm{e}$ inclinación de las flores $(r=-0.4792, p=0.0001)$. Así mismo, las flores producidas en las regiones apicales están expuestas a una alta densidad de estructuras $(r=0.4244$, $p=0.0008)$. 


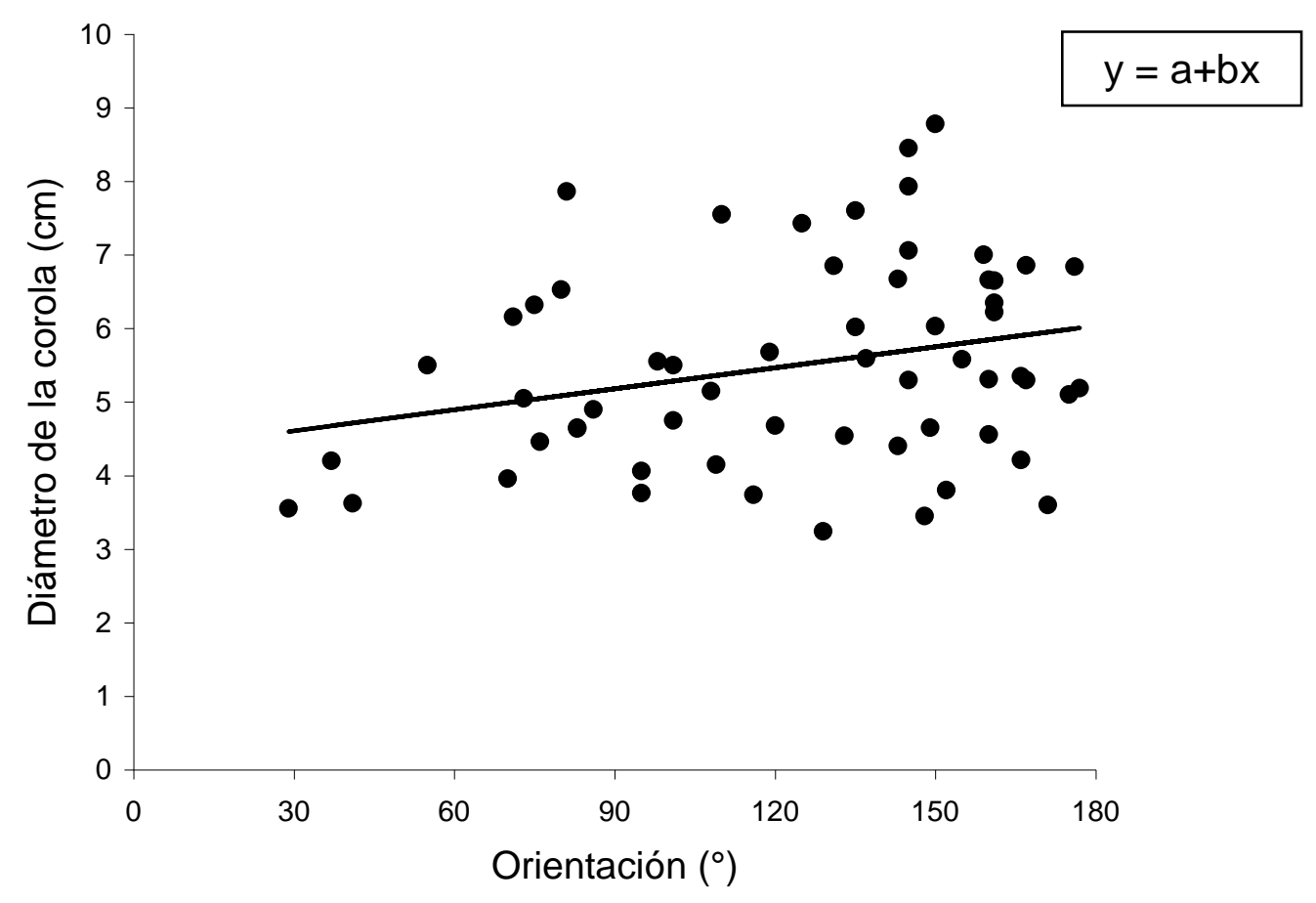

Figura 16. Relación entre la orientación y el diámetro de la corola de flores $(r=0.2704, p=0.0383)$ de $P$. weberi en San José Chichihualtepec, Oaxaca.

Al igual que en el caso de las 59 flores etiquetadas, la orientación de las 57 yemas florales colectadas para estimar el número de óvulos varió entre $0^{\circ}$ y $170^{\circ}$ por lo que nuevamente no fue necesario el uso de la estadística circular.

Así tenemos que existe una correlación positiva significativa entre la orientación de las yemas y el número de óvulos por ovario $(r=0.4165, p=0.0013)$. Esto sugiere que las yemas con orientación sur-sureste presentan el mayor número de óvulos por ovario (Figura 17). 


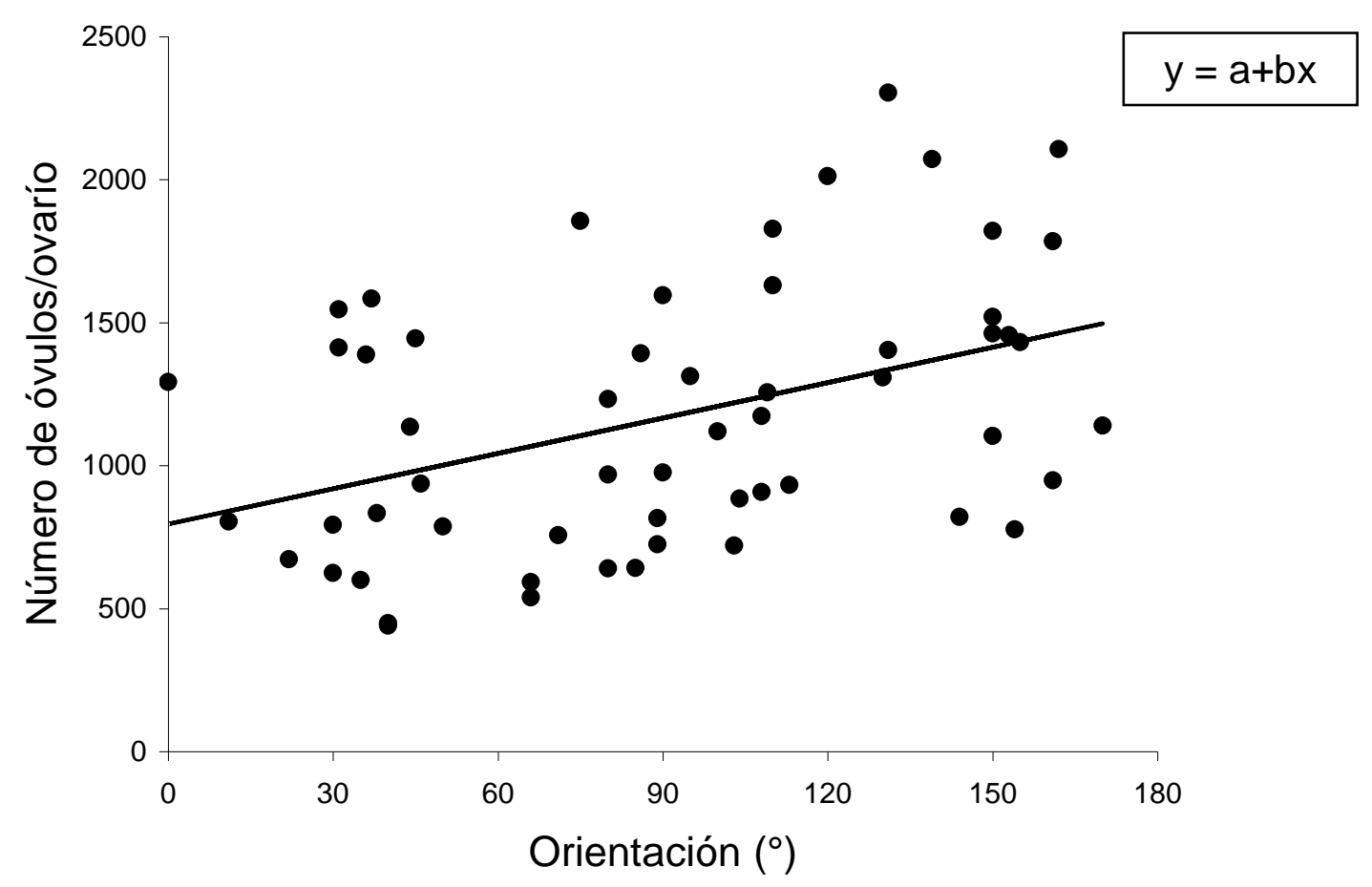

Figura 17. Relación entre la orientación y el número de óvulos/ovario en botones colectados $(r=0.4165, p=0.0013)$ de $P$. weberi en San José Chichihualtepec, Oaxaca.

El análisis de regresión logística múltiple reveló que la probabilidad de éxito en la producción de frutos (proporción de frutos exitosos) se incrementa con el diámetro medio y la orientación de las flores (Tabla 2). De acuerdo con lo anterior, flores con diámetro medio más amplio y con orientación sur-sureste son más exitosas en producir frutos. En este mismo sentido, el análisis de regresión múltiple reveló que el éxito reproductivo medido como el número de semillas por fruto (transformada a $\log _{10}$ $[x+1])$ es explicado de igual forma por el diámetro medio y orientación de las flores $\left(F_{2,56}=6.053, p=0.0042, R^{2}=0.18 ;\right.$ Tabla 3$)$. Este resultado sugiere que flores con 
diámetro medio más amplio y con orientación sur-sureste producen más semillas. Finalmente, el diámetro medio de la flor fue el único carácter que explicó la variación en la proporción de semillas producidas (modelo de regresión lineal simple; $b=$ $0.415, t=2.51, p=0.0150, r^{2}=0.10$ ). De nueva cuenta, este carácter tiene un efecto positivo sobre el éxito reproductivo medido como proporción de semillas.

El resto de las variables no tuvieron efecto significativo sobre el éxito reproductivo en ninguno de los análisis.

Tabla 2. Resultado del análisis de regresión logística múltiple (modelo reducido) utilizado para evaluar el efecto de los caracteres florales medidos in situ $(n=59)$ sobre la proporción de frutos exitosos de $P$. weberi en San José Chichihualtepec, Oaxaca.

\begin{tabular}{lcccc}
\hline Parámetros & Coeficiente & Error estándar & $\chi^{2}$ de Wald & $p$ \\
\hline Intersección & -11.885 & 5.053 & 5.532 & 0.0197 \\
Diámetro medio & 3.768 & 1.406 & 7.177 & 0.0074 \\
Orientación & 0.018 & 0.009 & 4.246 & 0.0391
\end{tabular}

Tabla 3. Resultado del análisis de regresión múltiple (modelo reducido) utilizado para evaluar el efecto de los caracteres florales medidos in situ $(n=59)$ sobre el número de semillas de $P$. weberi en San José Chichihualtepec, Oaxaca.

\begin{tabular}{lcccc}
\hline Parámetros & Coeficiente & Error estándar & $t$ & $p$ \\
\hline Intersección & -5.118 & 1.883 & -2.79 & 0.0072 \\
Diámetro medio & 2.064 & 0.664 & 3.11 & 0.0030 \\
Orientación & 0.009 & 0.004 & 2.20 & 0.0317 \\
\hline
\end{tabular}




\subsubsection{Experimento para evaluar el efecto del aroma como carácter floral y su relación con el éxito reproductivo}

Aunque el número de frutos producidos en el tratamiento con aroma "aumentado" (8) fue ligeramente mayor que en el tratamiento control (7) estas diferencias no fueron significativas $\left(\chi^{2}\right.$-pareada $\left.=0.1825, g . l .=1, p=0.6691\right)$.

De igual forma, el número promedio de semillas por fruto, la proporción de semillas producidas y el peso promedio de las semillas fue mayor en el tratamiento con aroma "aumentado". Sin embargo, las diferencias no fueron significativas (Tabla $4)$.

Tabla 4. Pruebas de $t$-pareadas de una cola de la comparación entre tratamientos del experimento para evaluar el efecto del aroma en el éxito reproductivo.

\begin{tabular}{lcccc}
\hline \multicolumn{5}{c}{ Tratamientos (media \pm D.E.) } \\
& Aroma "aumentado" & Control & $t$-pareada & $p$ \\
\hline $\begin{array}{l}\text { Número de } \\
\text { semillas/fruto }\end{array}$ & $611.2 \pm 124.0$ & $492.9 \pm 120.5$ & 1.1384 & 0.1421 \\
$\begin{array}{l}\text { Proporción de } \\
\text { semillas }\end{array}$ & $0.496 \pm 0.1$ & $0.368 \pm 0.1$ & 1.3963 & 0.0980 \\
$\begin{array}{l}\text { Peso promedio } \\
\text { de las semillas }\end{array}$ & $1.712 \pm 0.4$ & $1.452 \pm 0.4$ & 0.8907 & 0.1983 \\
\end{tabular}




\section{Discusión}

\section{Orientación de estructuras reproductivas}

Este estudio muestra que las estructuras reproductivas en Pachycereus weberi se producen preferentemente hacia el sur, en donde se presentan las temperaturas más altas en el día al igual que los valores más altos de humedad relativa durante la noche. Si bien no contamos con una explicación sobre el por qué el lado Sur presenta condiciones de humedad nocturna más benignas, resulta más que evidente que esta condición es favorable para especies con metabolismo CAM como $P$. weberi. Si la mayor humedad relativa en la cara Sur de las ramas durante la noche es una consecuencia de la mayor actividad respiratoria por la presencia de las flores, o si es consecuencia de la acumulación de vapor de agua derivado de las más altas temperaturas registradas durante el día, son hipótesis que requieren ponerse a prueba en futuros estudios.

Las hipótesis propuestas para explicar este patrón, reportado esencialmente para cactos extratropicales, plantean que la orientación hacia el ecuador garantiza las temperaturas óptimas para el desarrollo de las flores (Ehleringer et al., 1980; Nobel, 1981; Tinoco-Ojanguren y Molina-Freaner, 2000). Así por ejemplo, para especies como Carnegiea gigantea y Pachycereus pringlei, en el hemisferio Norte, y Copiapoa spp. y Trichocereus spp. en el hemisferio Sur, el incremento en las temperaturas alcanzadas por la orientación ecuatorial mejora el desarrollo de las flores al favorecer la actividad meristemática (Nobel, 1981). En este sentido, TinocoOjanguren y Molina-Freaner (2000) sugieren que la intercepción de Radiación 
Fotosintéticamente Activa (RFA), es el factor responsable de la orientación de las flores en $P$. pringlei en el Desierto Sonorense, suponiendo que la ganancia de carbono difiere entre costillas con diferente acimut y que no existe translocación entre ellas. Estos autores proponen que si la inducción de las aréolas para la producción de flores depende de la acumulación de carbohidratos, aquellas con la orientación más sureña serán capaces de acumular suficientes carbohidratos que permitan la producción y desarrollo de las flores. Así, una orientación sureña maximiza la productividad de las flores debido a una mayor captación de luz y mayor RFA (Gibson y Nobel, 1986).

Aunque $P$. weberi es un cacto con distribución intertropical, los resultados de este estudio sugieren que dicha especie se comporta como una especie extratropical y podría invocarse al mecanismo propuesto por Tinoco-Ojanguren y Molina-Freaner (2000) en P. pringlei para explicar la orientación ecuatorial de sus estructuras reproductivas.

Por otro lado, según varios autores, la orientación de las flores es un rasgo que ha evolucionado por presiones selectivas atribuidas a factores ambientales abióticos tales como la temperatura y precipitación (Huang et al., 2002; Patiño et al., 2002; Galen y Stanton, 2003; Ushimaru y Hyodo, 2005). Cabe destacar que además de la temperatura y radiación solar (factores abióticos), existen rasgos florales que pueden influir en el desarrollo diferencial de flores y frutos con distinta orientación. Tales rasgos florales incluyen la forma, el tamaño y calidad de la recompensa, que intervienen en la polinización y que podrían variar con la orientación, por lo tanto, 
influir en el éxito reproductivo en cuanto a producción de frutos y semillas (Patiño et al., 2002; Galen y Stanton, 2003; Ushimaru et al., 2006).

En este sentido, nuestra evidencia sugiere que la orientación de las flores es un caracter floral que se encuentra relacionado con la atracción de murciélagos en $P$. weberi, y por lo tanto, según Ushimaru y colaboradores (2006) es un caracter sujeto a evolución por selección natural.

\section{Relación entre los caracteres florales asociados al síndrome de polinización quiropterófilo en Pachycereus weberi.}

Los resultados de este estudio muestran que el diámetro de la corola es el único carácter floral medido in situ que se encuentra correlacionado significativamente con la orientación. Esto sugiere que flores con corolas más amplias se encuentran distribuidas preferencialmente hacia el sur, en donde se encuentran las condiciones microambientales óptimas para su desarrollo y crecimiento. Conner y Rush (1996) sugieren que el incremento en el tamaño de la corola y número de flores, favorece la tasa de visitas de polinizadores potenciales a las flores de varias especies de angiospermas, entre ellas destaca Raphanus raphanistrum, un rábano silvestre. Dichos autores mencionan que el tamaño de la corola es un rasgo floral que se encuentra correlacionado, en algunos casos con la producción de polen en flores. 
La orientación también se encontró correlacionada significativamente con el número de óvulos por ovario, lo cual nos sugiere que las yemas florales y flores que se encuentran orientadas hacia el sur, desarrollan una mayor producción de óvulos por ovario. Para explicar dicha producción diferencial de óvulos por ovario en $P$. weberi, también invocamos la hipótesis propuesta con anterioridad por TinocoOjanguren y Molina-Freaner (2000). Con base en este supuesto, podemos argumentar que el lado sur de las ramas de $P$. weberi presentan una mayor radiación solar (RFA), propiciando una mayor acumulación de fotosintatos en las costillas, favoreciendo el desarrollo y crecimiento de los tejidos que conformarán cada una de las partes florales que integrarán las flores en esta especie. Por lo tanto, nuestra evidencia indica que las flores dispuestas hacia el sur, serán capaces de producir una mayor cantidad de óvulos por ovario debido a las condiciones microambientales favorables para su producción y crecimiento.

Con respecto al resto de los caracteres florales medidos in situ, el diámetro de la base de las flores se encuentra correlacionado positivamente con la longitud y el diámetro medio. Esto nos sugiere que las flores con diámetros basales más amplios están asociados con flores más grandes, en cuanto a longitud y grosor medio, confiriéndoles probablemente un beneficio de resistencia mecánica ante los embates de las visitas de los murciélagos durante la apertura y cierre de sus corolas (antesis) (Faegri y van der Pijl, 1979; Stanton et al., 1986; Sutherland, 1986, 1987; Navarro et al., 2000). 
Por otra parte, ciertos caracteres florales se correlacionan negativamente debido al incremento de la densidad de estructuras reproductivas, como es el caso de la longitud, diámetro de la base de la flor e inclinación. Esto nos indica que el aumento de la densidad de estructuras reproductivas alrededor de la flor focal, implica una disminución de su tamaño, sugiriendo probablemente, un costo por la limitación de recursos dentro de individuos en $P$. weberi. Adicionalmente, la inclinación de la flor muestra una correlación negativa con la altura de su posición con respecto a la planta. Esto sugiere que las flores que se desarrollan en las regiones más apicales de las ramas tienden a crecer verticalmente, aludiendo un beneficio de la exposición vertical de la corola en el contexto en el cual se producen y desarrollan las flores en las ramas de Pachycereus weberi. Sin embargo, cabe resaltar que la densidad de estructuras reproductivas es sólo una variable de contexto que permite explicar el patrón en el cual se producen y desarrollan las flores en esta cactácea arborescente.

Algunos autores argumentan que la variabilidad en los caracteres florales implica costos-beneficios relacionados con la disponibilidad de recursos en la planta, los cuales pueden influir positiva o negativamente en su éxito reproductivo, en términos de producción de frutos y semillas (Cruden y Lyon, 1985; Dafni y Kevan, 1997; Bazzaz et al., 1987). Los resultados obtenidos en el presente trabajo, sugieren que dichos caracteres florales juegan un papel importante en la tasa de visita de murciélagos. No obstante, este estudio no midió la frecuencia de visitas de 
murciélagos a las flores de $P$. weberi y su relación con la variación de caracteres florales. Esta cuestión tendrá que responderse en estudios futuros.

A largo plazo, será útil estimar la tasa de visitas de los murciélagos a las flores de $P$. weberi con distribución preferencial y evaluar la atracción que podría representar dicho parche de recursos en estos polinizadores.

\section{Efecto de la variación de los caracteres florales sobre el éxito reproductivo de P. weberi}

El análisis de la variación de los caracteres florales sobre el éxito reproductivo en Pachycereus weberi, revelan que la probabilidad de éxito en la producción de frutos y semillas, aumenta con el diámetro medio y la orientación de las flores. Adicionalmente, nuestra evidencia muestra que el diámetro medio de las flores fue el único carácter floral (asociado al síndrome quiropterófilo) que explicó la variación en cuanto a la proporción de semillas producidas de $P$. weberi. Esto nos sugiere que el diámetro medio de la flor tiene un efecto positivo sobre el éxito reproductivo de esta cactácea columnar. Este resultado es de destacar considerando que es uno de los escasos estudios que muestran la selección de rasgos florales por murciélagos y sus consecuencias en el éxito reproductivo en cactáceas. En este sentido, algunos autores apuntan que la orientación y el tamaño de las flores son atributos determinantes en el éxito reproductivo de angiospermas (Faegri y van der Pijl, 1979; Stanton et al., 1986; Sutherland, 1986, 1987; Fleming y Sosa, 1994; Navarro et al., 2000). Por tal motivo, nuestra evidencia sugiere que los caracteres o atributos 
florales mencionados anteriormente, tienen un efecto en el éxito reproductivo medido en su paso exitoso de flor a fruto, así como también, en cuanto al número de semillas producido en cada fruto y la correspondiente proporción de las mismas. Sin embargo, también ha sido argumentado que los factores abióticos tales como la temperatura, la radiación solar y la precipitación, por citar algunos, son los responsables de la variabilidad existente en la morfología de las plantas. Dichos factores han determinado la evolución de los rasgos florales en las angiospermas, entre ellos destacan el color de las flores, el tamaño, la simetría, la orientación y las esencias florales (Sutherland, 1986; Totland, 1996; von Helversen y von Helversen, 1999; Galen y Stanton, 2003; Ushimaru et al., 2006).

\section{Experimento para evaluar el efecto del aroma como carácter floral y su relación con el éxito reproductivo}

Existen evidencias sobre la importancia de las señales sensoriales que reciben los polinizadores a grandes distancias, indicándoles una fuente abundante de recursos alimenticios (néctar y/o polen) (Faegri y van der Pijl, 1979; Knudsen y Tollsten, 1995; Tschapka y Dressler, 2002; Raguso, 2006; Muchhala, 2007). Por tal motivo, algunos autores han implementado diseños experimentales para manipular (es decir, "aumentar") el aroma natural de las flores en algunas especies de angiospermas y, evaluar de esta forma, el efecto del aroma sobre la atracción de polinizadores, contabilizando la tasa de visita a dichas flores y, por consiguiente, evaluar el éxito reproductivo (exportación de polen, proporción de frutos exitosos y/o 
proporción de semillas) en estas plantas (Campbell, 1989; Miyake y Yafuso, 2003; Raguso, 2006; Ashman et al., 2005).

Los resultados de este estudio indican que el número de frutos producidos, el número y peso de las semillas por fruto, además de la proporción de las mismas, fueron mayores en el tratamiento experimental con aroma "aumentado" en comparación con el tratamiento control, sin embargo, las diferencias no fueron significativas. Estos resultados experimentales no son concluyentes en poner a prueba la importancia del aroma como carácter floral en la atracción de murciélagos y su efecto sobre el éxito reproductivo de $P$. weberi. El reducido tamaño de muestra ( $n=10$ botones florales para cada tratamiento en 10 individuos de $P$. weberi), probablemente careció de poder de detección.

Futuros estudios que consideren el incremento del tamaño de muestra, podrían aportar información suficiente y relevante para probar, experimentalmente, el posible efecto del aroma de las flores sobre el éxito reproductivo, en términos de producción de frutos y semillas en $P$. weberi. No obstante, las esencias florales rara vez actúan solas. Por lo regular se encuentran asociadas con otras señales sensoriales, tales como el color, la forma y simetría de las flores, la textura del néctar que, seguramente son utilizadas por los visitantes florales (Raguso, 2006).

En el caso de las cactáceas columnares, el presente estudio intentó aportar información que, a largo plazo permitirá profundizar y esclarecer en futuros estudios, la contribución de los aromas florales en la compleja interacción planta-polinizador y 
el efecto que pudiera tener en el éxito reproductivo de estas plantas, emblemáticas de las zonas áridas y semiáridas del continente americano.

\section{CONCLUSIÓn}

Pachycereus weberi es una cactácea arborescente presente en la Reserva de la Biosfera de Tehuacán-Cuicatlán, entre los estados de Puebla y Oaxaca, México. Sus estructuras reproductivas presentan un patrón de distribución preferencial hacia el sur, coincidiendo dicho acimut con los valores de temperatura más altos durante el día y los porcentajes de humedad relativa más altos durante la noche. En este sentido, dichas condiciones microambientales (temperatura y humedad relativa), favorecen una mayor absorción de la Radiación Fotosintéticamente Activa (RFA) y por consiguiente, una mayor captura de $\mathrm{CO}_{2}$ durante la noche (metabolismo CAM) en las caras de las ramificaciones con orientación sur, esto propicia una acumulación de fotosintatos, los cuales son los responsables del desarrollo y crecimiento de yemas florales, flores y frutos en $P$. weberi.

La orientación preferencial sur le confiere beneficios a esta planta en cuanto a los procesos de polinización, dado que la orientación de las flores se encuentra correlacionada significativamente con un carácter floral asociado al síndrome de polinización quiropterófilo: el diámetro de la corola. Por otro lado, la orientación también tiene un efecto positivo en el número de óvulos por ovario producidos por las flores sureñas. Estas evidencias nos sugieren que dichos caracteres florales son muy atractivos para los murciélagos, quienes se benefician de las recompensas florales 
(néctar y/o polen) que esta cactácea columnar les ofrece a cambio de visitar sus flores.

También en este estudio se muestra que hay un efecto de la orientación y el diámetro medio de las flores de P.weberi sobre el éxito reproductivo en términos de proporción de frutos exitosos y proporción de semillas. Por lo tanto, una ligera variación en el tamaño de la corola, cantidad de recompensas florales (néctar y/o polen), longitud de las flores y/o ángulo de inclinación de las flores, entre otros más, podría afectar la frecuencia de visitas de los murciélagos nectarívoros, disminuyendo la producción de frutos y semillas en $P$. weberi.

Esto nos indica que la orientación de las flores y la variabilidad de los caracteres florales tienen efectos positivos en la polinización y en los eventos post-polinización.

El presente estudio subraya la importancia de la selección de rasgos florales por los murciélagos y sus consecuencias en el éxito reproductivo, en su paso de flor a fruto en esta cactácea columnar, presente en la reserva de la biosfera de TehuacánCuicatlán. 


\section{Bibliografía}

Alcorn, S.M., McGregor, S.E. y Olin, G. 1962. Pollination requirements of the organpipe cactus. Cactus and Succulent Journal 34: 134-138.

Arias, S., Gama, L.S. y Guzmán, C. L. U. 1997. Flora del Valle de TehuacánCuicatlán. Fascículo 14. Cactaceae A. L. Juss. UNAM. México, D.F. 185 p.

Arias-Cóyotl, E., Stoner, K. E. y Casas, A. 2006. Effectiveness of bats as pollinators of Stenocereus stellatus (Cactaceae) in wild, managed in situ and cultivated populations in La Mixteca Baja, Central Mexico. American Journal of Botany 96 (11): $1675-1683$.

Arita, H. T. y Martínez del Río, C. 1990. Interacciones Flor-murciélago: un enfoque zoocéntrico. Instituto de Biología Publicaciones especiales 4. UNAM. México, D.F.

Ashman, T. L., Cole, D.H., Bradburn, M., Blaney, B. y Raguso, R. A. 2005. The scent of a male: the role of floral volatiles in pollination of a gender dimorphic plant. Ecology 86 (8): 2099-2105.

Bazzaz, F.A., Chiariello, N.R., Coley, P.D. y Pitelka, F. L. 1987. Allocating resources to reproduction and defense. Plant Phisiological and Ecology 37 (1): 58-67.

Bravo-Hollis, H. y Sánchez-Mejorada, H. 1978. Las cactáceas de México. Volumen I. Universidad Nacional Autónoma de México. México, D.F. 
Bustamante, E. y Búrquez, A. 2005. Fenología y biología reproductiva de las cactáceas columnares. Cactáceas y Suculentas Mexicanas 50 (3): 68-88.

Campbell, D. R. 1989. Measurements of selection in a hermaphroditic plant: variation in male and female pollination success. Evolution 43 (2): 318-334.

Casas, A., Valiente-Banuet, A., Rojas-Martínez, A. y Dávila, P. 1999. Reproductive biology and the process of domestication of the columnar cactus Stenocereus stellatus in Central, Mexico. American Journal of Botany 86 (4): 534-542.

Conner, J.K. y Rush, S. 1996. Effects of flower size and number on pollinator visitation to wild radish, Raphanus raphanistrum. Oecologia 105 (4): 509-516.

Córdova-Acosta, E., Figueroa-Castro, D.M., Vite, F. y Valverde, P.L. 2009. Pachycereus weberi (J. M. Coulter) Backeb. Cactáceas y Suculentas Mexicanas 54 (4): 128.

Cruden, R. W. y Lyon, D. L. 1985. Patterns of biomass allocation to male and female and functions in plants with different mating systems. Oecologia 66 (2): 299306.

Dafni, A. y Kevan, P.G. 1997. Flower size and shape: implication in pollination. Israel Journal of Plant Science 45: 201-212.

Ehleringer, J.R., Money, H. A., Gulmon, S.L. y Rundel, P. 1980. Orientation and it's consequences for Copiapoa (Cactaceae) in Atacama Desert. Oecologia 46: 63-67. 
Faegri, K. y van der Pijl, L. 1979. The principles of pollination ecology. Oxford Pergamon Press. Nueva York, Estados Unidos de América.

Feinsinger, P. 1983. Coevolution and pollination, pp. 282-310. En: D. J. Futuyma y M. Slatkin (eds.) Coevolution. Sinauer, Sunderland, Massachussetts, Estados Unidos de América.

Fenster, C.B., Armbruster, W.S., Wilson, P., Dudash, M.R. y Thomson, J.D. 2004. Pollination syndromes and floral specialization. Annual Review of Ecology, Evolution and Systematics 35: 375-403.

Fleming, T. H. 1993. Plant-visiting bats. American Scientist 81: 460-467.

Fleming, T.H. y Sosa, V. 1994. Effects of nectarivorous and frugivorous mammals on reproductive success of plants. Journal of Mammalogy 75 (4): 845-851.

Fleming, T.H., Sahley, C.T., Holland, J.N., Nason, J.D. y Hamrick, J.R. 2001. Sonoran desert columnar cacti and the evolution of generalized pollination system. Ecological Monographs 71 (4): 511-530.

Galen, C. 1989. Measuring pollinator-mediated selection on morphometric floral traits: Bumblebees and the alpine sky pilot, Palemonium viscosum. Evolution 43 (4): 882-890.

Galen, C. y Stanton, M.L. 2003. Sunny-side up: flower heliotropism as a source of parental effects on pollen quality and performance in the snow buttercup, 
Ranunculus adoneus (Ranunculaceae). American Journal of Botany 90: 724729.

Geller, G.N. y Nobel, P.S. 1986. Branching patterns of columnar cacti: influences on PAR interception and CO2, uptake. American Journal of Botany 73: 11931200.

Grant, V. y Grant, K. A. 1979. The pollination spectrum in the Southwestern American cactus flora. Plant Systematics and Evolution 133: 29-37.

Gibson, A.C. y Nobel, P.S. 1986. The cactus primer. Harvard University Press, Cambridge, Massachussetts, Estados Unidos de América.

von Herversen, D. y von Helversen, O. 1999. Acoustic guide in bat-pollinated flowers. Nature 398: 759-760.

Hintze, J. 2001. NCSS and PASS. Number Cruncher Statistical Systems (Kaysvill, Utah).

Howell, D.J. 1974. Bats and pollen: Physiological aspects of the syndrome of chiropterophily. Comparative Biochemistry and Physiology 48 (2 A): 263-276.

Huang, S.Q., Takahashi, Y. y Dafni, A. 2002. Why does the flower of Pulsatilla cernua (Ranunculaceae) bend during anthesis?. American Journal of Botany 89 (10): 1599-1603. 
Johnson, D.S. 1924. The influence of insolation on the distribution and on developmental sequence of flowers of giant cactus of Arizona. Ecology 5: 7082.

Knudsen, J.D. y Tollsten, L. 1995. Floral scent in bat-pollinated plants: a case of convergent evolution. Botanical Journal of the Linnean Society 119 (1): 45-57.

Kovach, W.L. 2004. ORIANA v 2.02. Kovach Computing Services. Pentraeth, Gales, Reino Unido (U.K.).

Kovach, W.L. 2010. XLStat v. 2010. Kovach Computing Services. Pentraeth, Gales Reino Unido (U.K.).

Medel, R., Botto-Mahan, C. y Kalin-Arroyo, M. 2003. Pollinator mediated selection on the nectar guide phenotype in the andean monkey flower, Mimulus luteus. Ecology 84 (7): 1721-1732.

Mendenhall, W. y Sincich, T. 2003. A second course in statistics: regression analysis. Pearson Education, Upper Saddle River, NJ.

Miyake, T. y Yafuso, M. 2003. Floral scents affect reproductive success in flypollinated Alocasia odora (Araceae). American Journal of Botany 90: 370-376.

Moller, A.P. y Eriksson, M. 1995. Pollinator preference for symmetrical flowers and sexual selection in plants. Oikos 73: 15-22.

Moran, R. 1962. Visitors to the flowers of Pachycereus pringlei. Cactus and Succulent Journal 17: 21. 
Muchhala, N. 2007. Adaptive trade-off in floral Morphology mediates specialization for flowers pollinated by bats and hummingbirds. The American Naturalist 169: 494-504.

Nassar, J.M., Ramírez, N. y Linares, O. 1997. Comparative pollination biology of Venezuelan columnar cacti and the role of nectar-feeding bats in their sexual reproduction. American Journal of Botany 84 (8): 918-927.

Navarro, L., Rosas, C. y Ayensa, G. 2000. Recompensas florales y éxito reproductivo. Portugaliae Acta Biological 19: 121-126.

Neter, J., Kutner, M.H., Nachtsheim, C.J. y Wasserman, W. 1996. Applied linear regression models. Irwin, Chicago, IL, Estados Unidos de América.

Nobel, P.S. 1980. Morphology, nurse plants and minimum apical temperatures for young Carnegiea gigantea. Botanical Gazete 141: 188-191.

Nobel, P.S. 1981. Influences of photosynthetical active radiation on cladode orientation, stem tilting and height of cacti. Ecology 62: 982-990.

Nobel, P.S. 1986. From and orientation in relation to PAR interception by cacti and agaves. En: On the economy of plant form and function. Editado por: T. J. Givnish. Cambridge University Press, Cambridge, U. K. P. 83-103.

Nobel, P.S. 1994. Remarkable agaves and cacti. Oxford University Press, New York, Estados Unidos de América. 
Nobel P.S. y Loik, M.E. 1999. Form and function of cacti. En: R. H. Robichaux (ed.) Ecology of Sonoran Desert plants and plants communities. The University of Arizona Press, Tucson, P. 143-163.

Patiño, S., Jeffree C. y Grace, J. 2002. The ecological role of orientation in tropical convolvulaceous flowers. Oecologia 130: 373-379.

van der Pijl, L. 1961. Ecological aspects of flower evolution. II. Zoophilous flower class. Evolution 15: 44-59.

Raguso, R.A. 2004. Flowers as sensory billboards: progress towards an integrated understanding of floral advertisement. Plant Biology 7: 434-440.

Raguso, R.A. 2006. Behavioral responses to floral scent: experimental manipulations and the interplay of sensory modalities. En: Biology of Floral scent. Natalia Dudareva y Eran Pichersky (eds.). P. 297-318. CRS Press Taylor and Francis Group. Boca Raton, Florida, Estados Unidos de América.

Rufibach, K. 2011. Assessment of paired binary data. Skeletal Radiology 40: 1 - 4.

Rundel, P.W. 1974. Trichocereus in the Mediterranean zone of central Chile. Cactus Succulent Journal 46: 86-88.

Rzedowski, L. 1978. Vegetación de México. Limusa, México, D.F.

Sahley, C.T. 2001. Vertebrate pollination, fruit production and pollen dispersal of Stenocereus thurberi (Cactaceae). The Southwestern Naturalist 46 (3): 261271. 
SAS Institute, Inc. 1999. SAS/STAT User's Guide, v. 8.2. Cary, NC:SAS Institute.

Stanton, M.L., Snow, A.A. y Handel, S.N. 1986. Floral evolution: attractiveness to pollinators increases male fitness. Science 232: 1625-1627.

Stanton, M.L. y Galen, C. 1989. Consequences of flower heliotropism for reproduction in an alpine buttercup (Ranunculus adoneus). Oecologia 78: 477-485.

Stebbins, G.L. 1970. Adaptive radiation of reproductive characteristics in angiosperms. I. Pollination mechanisms. Annual Review of Ecology and Systematics 1: 307-326.

Sutherland, S. 1986. Floral sex ratios, fruit-set and resource allocation in plants. Ecology 67 (4): 991-1001.

Sutherland, S. 1987. Why hermaphroditic plants produce many more flowers than fruits: experimental test with Agave mckelveyana. Evolution 41 (4): 750-759.

Tadey, M. y Aizen, M.A. 2001. Why do flowers of a hummingbird-pollinated mistletoe face-down?. Functional Ecology 15: 782-790.

Tinoco-Ojanguren, C. y Molina-Freaner, F. 2000. Flower orientation in Pachycereus pringlei. Canadian Journal of Botany 78: 1489 -1494.

Totland, Ö. 1996. Flowers heliotropism in an alpine population of Ranunculus acris (Ranunculaceae): effects on flowers temperature, insect visitation and seed production. American Journal of Botany 83 (4): 452-458. 
Tschapka, M. y Dressler, S. 2002. Chiropterophily: on bat-flowers and flowers-bats. Curti's Botanical Magazine 19 (2): 114-125.

Ushimaru, A., Kawase, D. y Imamura, A. 2006. Flowers adaptively face down-slope in 10 forest-floor herbs. Functional Ecology 20: 585-591.

Valiente-Banuet, A., Arizmendi, M. C., Rojas-Martínez, A. y Domínguez-Canseco, L. 1996. Ecological relationships between columnar cacti and nectar-feeding bats in México. Journal of Tropical Ecology 12: 103-119.

Valiente-Banuet, A., Rojas-Martínez, A., Casas, A., Arizmendi, M.C. y Dávila, P. 1997. Pollination biology of two winter-blooming giant columnar cacti in the Tehuacán Valley, Central Mexico. Journal of Arid Environments 37: 331-341.

Valverde, P.L., Vite, F. y Rodríguez-Salinas, Y. 2006. Evaluación de la función termorreguradora del pseudocefalio en Cephalocereus columna-trajani (Kart. Ex Pfeiff.) K. Schum. Cactáceas y Suculentas Mexicanas 51: 52-63.

Valverde, P.L., Vite, F., Pérez-Hernández, M.A. y Zavala-Hurtado, J.A. 2007. Stem tilting, pseudocephalium orientation, and stem allometry in Cephalocereus columna-trajani along a short latitudinal gradient. Plant Ecology 188: 17-27.

Yeaton, R.I., Karban, R. y Wagner, H.B. 1980. Morphological growth patterns of saguaro (Carnegiea gigantea: Cactaceae) on flatsand slopes in organ pipe cactus National Monument, Arizona. Southwestern Naturalist 25: 334-349. 
Zavala-Hurtado, J.A., Vite, F. y Ezcurra, E. 1998. Stem tilting and pseudocephalium orientation in Cephalocereus columna-trajani (Cactaceae): a functional interpretation. Ecology 79: 340-348.

Zar, J.H. 1999. Biostatistical Analysis. Prentice Hall, Upper Saddler River. 